\title{
Model Reduction in Flexible-Aircraft Dynamics with Large Rigid-Body Motion
}

\author{
Henrik Hesse* and Rafael Palacios ${ }^{\dagger}$ \\ Imperial College, London SW7 2AZ, United Kingdom
}

\begin{abstract}
This paper investigates the model reduction, using balanced realizations, of the unsteady aerodynamics of maneuvering flexible aircraft. The aeroelastic response of the vehicle, which may be subject to large wing deformations at trimmed flight, is captured by coupling a displacement-based, flexible-body dynamics formulation with an aerodynamic model based on the unsteady vortex lattice method. Consistent linearization of the aeroelastic problem allows the projection of the structural degrees of freedom on a few vibration modes of the unconstrained vehicle, but preserves all couplings between the rigid and elastic motions and permits the vehicle flight dynamics to have arbitrarily-large angular velocities. The high-order aerodynamic system, which defines the mapping between the small number of generalized coordinates and unsteady aerodynamic loads, is then reduced using the balanced truncation method. Numerical studies on a representative high-altitude, long-endurance aircraft show a very substantial reduction in model size, by up to three orders of magnitude, that leads to model orders (and computational cost) similar to those in conventional frequency-based methods but with higher modeling fidelity to compute maneuver loads. Closed-loop results for the Goland wing finally demonstrate the application of this approach in the synthesis of a robust flutter suppression controller.
\end{abstract}

\section{Nomenclature}

$\begin{array}{ll}\text { Symbols } & \\ \mathcal{C} & \text { global tangent damping matrix } \\ \mathcal{K} & \text { global tangent stiffness matrix } \\ \mathcal{M} & \text { global tangent mass matrix } \\ q & \text { generalized displacements in modal basis } \\ Q_{\text {ext }} & \text { global vector of external forces, } \mathrm{N} \\ t & \text { physical time, } \mathrm{s} \\ u & \text { input vector } \\ v & \text { inertial translational velocity of the body-fixed frame, } \mathrm{m} / \mathrm{s} \\ W_{c} & \text { controllability Gramian } \\ W_{o} & \text { observability Gramian } \\ x & \text { state vector of a linear system identified by subscripts } \\ y & \text { output vector } \\ \beta & \text { vector of global translational and rotational velocities } \\ \Gamma & \text { circulation strength of a vortex ring, } \mathrm{m}^{2} / \mathrm{s} \\ \eta & \text { vector of nodal displacements and rotations } \\ \Theta & \text { Euler angles, rad } \\ \nu & \text { global displacements and rotations as time integral of } \beta \\ \Phi & \text { matrix of mode shapes }\end{array}$

*Graduate Student, Department of Aeronautics, AIAA Student Member.

${ }^{\dagger}$ Senior Lecturer, Department of Aeronautics (Contact author: rpalacio@imperial.ac.uk), AIAA Member. 
$\begin{array}{ll}\chi & \text { coordinates of the aerodynamic lattice, } \mathrm{m} \\ \omega & \text { inertial angular velocity of the body-fixed frame, } \mathrm{rad} / \mathrm{s}\end{array}$

Subscripts

$A$

$A S$

$b$

$f$

$R$

$S$

$w$

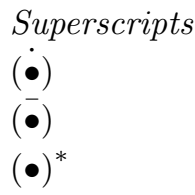

aerodynamic

aeroelastic, including rigid-body and structural states

bound, corresponding to lifting surface

flutter

rigid-body degrees of freedom

structural degrees of freedom

wake

derivatives with respect to time, $t$

small perturbations around an equilibrium

conjugate transpose of a matrix

\section{Introduction}

Low-speed unsteady aerodynamic loads have traditionally been obtained in aeroelasticity using the Doublet Lattice Method (DLM), ${ }^{1}$ which computes the aerodynamic influence coefficients for a few structural vibration modes over a range of reduced frequencies and flight conditions. This then requires some curvefitting, usually through rational-function approximations, ${ }^{2,3}$ to obtain time-domain state-space models. Even though this approach has been successfully applied to obtain transient responses and in controller design, it still requires suitable parameters to be set for the transformation to time domain, which can make this approach cumbersome. ${ }^{4}$ Furthermore, DLM-based solutions assume small normal displacements of the lifting surfaces, which may be no longer valid in high-load situations or for very flexible wings.

Time-domain aerodynamic models, such as the Unsteady Vortex Lattice Method (UVLM), provide a more flexible alternative to frequency-domain methods. They evaluate loads on the instantaneous deformed geometry and therefore can be coupled directly to geometrically-exact flexible-body dynamic models of the maneuvering aircraft. This improved kinematic characterization in the structural dynamics also removes the need for a priori assumptions on the inertial coupling between the vehicle structural and flight-dynamic response, as is done in the mean-axes approximation. ${ }^{5}$ It was shown in previous work ${ }^{6,7}$ that such decoupling of the system variable may have a significant impact on the predicted vehicle dynamic gust and maneuver loads.

However, time-domain aeroelastic solutions expressed in terms of the instantaneous circulation in all aerodynamic panels are still computationally expensive for controller design and flight dynamic simulation where lower-order models would be desired. This can be achieved through model reduction of the linearized unsteady aerodynamic system (around a reference nonlinear equilibrium condition). Inspired by the effective modal projection of large structural dynamics problems onto a few vibration modes of the structure, Hall ${ }^{8}$ demonstrated that the governing equations of a time domain unsteady vortex lattice model can be formulated as a generalized eigenvalue problem to produce a very compact, reduced-order aerodynamic model. Computing eigenvalues becomes however computationally expensive for large systems. This problem has been addressed in the fluid dynamics community using the proper orthogonal decomposition (POD), also known as the Karhunen-Loève expansion, ${ }^{9}$ to produce a basis function of the system based on sample data of the flow. ${ }^{10,11}$ Dowell et al..$^{12}$ and Lucia et al. ${ }^{13}$ both provide in-depth reviews of the application of POD to high-order linear and nonlinear computational fluid dynamic problems.

The resulting basis functions, obtained through either eigenanalysis or POD, accurately capture the internal dynamics of the system, but a large number of modes (not only lightly-damped ones) may be required to effectively represent the particular input/output mapping in a typical aeroelastic problem. This has been demonstrated by Rule et al. ${ }^{14}$ for the unsteady vortex lattice method. Those authors propose an alternative model reduction method, adopted from the controls community, to obtain a reduced-order aerodynamic model that is optimal in terms of the particular transmission path between the system inputs and outputs. 
Balanced realizations ${ }^{15,16}$ are especially useful in unsteady aerodynamics, where a large number of states is used to transmit information from a comparably small number of inputs (geometry of the structure) to a small number of outputs (unsteady aerodynamic forces). Balanced states that have little contribution to a particular input/output mapping can be efficiently discarded. ${ }^{12}$ The balanced model reduction approach has already been demonstrated for aeroelastic systems on a $2 \mathrm{D}$ pitch-plunge problem ${ }^{14}$ and on a full linear aircraft model to obtain the flight loads following wake vortex encounters. ${ }^{17}$

The performance of balanced realization method is strongly linked to the number of system inputs and outputs, ${ }^{18}$ e.g. the number of elastic and rigid-body degrees of freedom (DoF) in an aeroelastic problem. Hence, this work will propose a model reduction approach for an integrated flexible-aircraft formulation which allows us to control the number of in-/outputs of the aerodynamic system by projecting the flexible-body dynamics part of the coupled system on a small modal basis to reduce the number of generalized coordinates and aerodynamic loads. This has been preferred to a direct system identification through step responses, as done recently by Haghighat et al., ${ }^{19}$ since the balancing approach uses the state-space structure of the original model in the reduction process. To model the dynamics of maneuvering flexible aircraft, previous work by the authors ${ }^{6}$ demonstrated a consistent linearization of the structural DoF in nonlinear flexible-aircraft dynamics problems. This provides a formulation which allows projection of the nonlinear flexible-body dynamics subsystem onto the vibration modes of the unconstrained structure at any reference condition while keeping the nonlinearity in the rigid-body dynamics equations. The linearization of the structural DoF is consistent such that all couplings between rigid-body and structural dynamics due to gyroscopic motions are preserved, which was found to have a significant impact on the transient loads of the structure. ${ }^{6}$ Coupled with a 3D UVLM, ${ }^{4}$ this nonlinear modal approach has been demonstrated for the dynamic analysis of maneuvering flexible aircraft with possibly large, geometrically-nonlinear trim deformations. ${ }^{7}$

Continuing on those studies, this work will investigate the application of balanced truncation on the aerodynamics of the transient dynamic analysis of maneuvering flexible aircraft. Under the assumption of small elastic deformations, the unsteady aerodynamic loads are computed using the linearized version of the UVLM, which has been implemented by Murua et al. ${ }^{4}$ and shown to be very suitable for the stability analysis of flexible aircraft ${ }^{4,7}$ and control design. ${ }^{20}$ For maneuvering aircraft, however, the underlying rigid-body dynamics equations of motion (EoM) are nonlinear and we will focus in this work on balanced truncation of the (linear) unsteady aerodynamic subsystem only whereas the nonlinear flexible-body dynamics are projected onto a small number of dominant vibration modes of the unconstrained structure. This reduces the number of generalized structural coordinates and aerodynamic loads - the inputs and outputs of the aerodynamic system - which is necessary for an effective reduction of the balanced aerodynamic subsystem. ${ }^{18}$ The resulting integrated aeroelastic framework is of very low order, but captures the 3D unsteady features of the flow and the tight coupling between the nonlinear flight dynamics of the aircraft and the structural wing deformations around a geometrically nonlinear static aeroelastic equilibrium.

\section{Flexible-Aircraft Dynamics Model}

This section describes the aerodynamic and flexible-body dynamic models used to compute the dynamics of flexible aircraft. The underlying geometrically nonlinear description of the flexible-aircraft dynamics can be found in previous papers ${ }^{6,21}$ and here we only focus on aspects of the linearization in both models, which will form the basis for model reduction of the coupled system in the next section.

\section{II.A. Unsteady Vortex Lattice Method}

The unsteady aerodynamic loads in this work are obtained using the linearized form of the unsteady vortex lattice method developed by Murua et al. ${ }^{4}$ and based on the description by Hall. ${ }^{8}$ In the UVLM, vortex ring quadrilateral elements are used to discretize both lifting surfaces and their wakes. Each surface (bound) vortex ring has an associated circulation strength, $\Gamma_{k}$, and a collocation point, at which the impermeability boundary condition is satisfied. To obtain the state-space form of the UVLM, the governing equations are linearized on a frozen aerodynamic geometry ${ }^{4,8}$ around the aircraft trim condition with possibly large wing deformations and non-planar wake.

The change in circulation strengths of the vortex rings around this steady-state solution is then given in discrete time as

$$
\Delta \Gamma^{n+1}=A_{A} \Delta \Gamma^{n}+B_{A} \Delta u_{A}^{n},
$$


where superscripts $n$ and $n+1$ refer to the current and next time steps and the subscript $A$ refers to the linear time-invariant (LTI) aerodynamics. The state vector, $\Gamma$, and inputs, $u_{A}$, are given by

$$
\Gamma=\left[\begin{array}{lll}
\Gamma_{b}^{\top} & \Gamma_{w}^{\top} & \dot{\Gamma}_{b}^{\top}
\end{array}\right]^{\top}, \quad \text { and } \quad u_{A}=\left[\begin{array}{lll}
\chi_{b}^{\top} & \dot{\chi}_{b}^{\top} & w_{g}^{\top}
\end{array}\right]^{\top},
$$

with the bound (surface) and wake circulation strengths, $\Gamma_{b}$ and $\Gamma_{w}$, respectively. The coordinates and timederivatives of the bound aerodynamic grid, $\chi_{b}$ and $\dot{\chi}_{b}$, respectively, can account for small elastic deformations of the lifting surfaces as well as the rigid-body velocities and orientation of the vehicle. The time-derivative of the aerodynamic grid, $\dot{\chi}_{b}$, is also included in Eq. (2) to account for deployment of control surfaces. Finally, $w_{g}$ refers to gust inputs.

The unsteady aerodynamic loads, which result from the vorticity distribution at each time step and are written in general as $\Delta y_{A}$, can be computed using the unsteady Bernoulli equation. ${ }^{4}$ This will be written in symbolic form as

$$
\Delta y_{A}^{n}=C_{A} \Delta \Gamma^{n}+D_{A} \Delta u_{A}^{n} .
$$

where the system feedthrough accounts for small changes in orientation of the aerodynamic loads. The resulting aerodynamic state-space system can be tightly-coupled with the flexible-body dynamics model, which is discussed next.

\section{II.B. Flexible-Body Dynamics Model}

The flexible vehicle will be modeled using composite beam elements on a moving (body-attached) frame of reference. Starting from a geometrically nonlinear displacement-based formulation, ${ }^{22,23}$ the elastic DoF are the displacements and rotations at the element nodes, which have been linearized using perturbation methods. ${ }^{6}$ This consistent linearization is done around a static equilibrium (trimmed aircraft in forward flight) with possibly large elastic deformations, which will be referred to as $\eta$. A detailed derivation of the linearization can be found in previous work. ${ }^{6,7}$ The resulting set of perturbation equations

$$
\mathcal{M}(\eta)\left\{\begin{array}{c}
\ddot{\bar{\eta}} \\
\dot{\beta}
\end{array}\right\}+\overline{\mathcal{C}}(\eta, \beta)\left\{\begin{array}{l}
\dot{\bar{\eta}} \\
\beta
\end{array}\right\}+\overline{\mathcal{K}}(\eta, \beta)\left\{\begin{array}{l}
\bar{\eta} \\
0
\end{array}\right\}=\bar{Q}_{\text {ext }}(\bar{\eta}, \dot{\bar{\eta}}, \beta, \zeta),
$$

accurately describe the structural dynamics of relatively-stiff aircraft undergoing arbitrary maneuvers with (not-necessarily small) rigid-body velocities, $\beta(t)$, resulting in small elastic deformations, $\bar{\eta}(t)$. The damping and stiffness matrices, $\overline{\mathcal{C}}$ and $\overline{\mathcal{K}}$, respectively, originate from the perturbation of the discrete gyroscopic and elastic forces of the geometrically nonlinear description and together with the mass matrix, $\mathcal{M}$, account for all coupling between the structural and rigid-body dynamics of the vehicle. It is important to note that the mass matrix $\mathcal{M}$ only depends on the static deformations, $\eta$, to account for a change in rotational inertia, but the damping and stiffness matrices are also functions of the instantaneous rigid-body velocity, $\beta$. See previous work by the authors ${ }^{6}$ for a detailed derivation of each of these matrices. The input from the aerodynamic model is accounted for in the external forcing term, $\bar{Q}_{\text {ext }}$, expressed at time $t^{n}=n \cdot \Delta t$ as

$$
\bar{Q}_{\text {ext }}\left(t^{n}\right)=\Delta y_{A}^{n}+\bar{Q}_{\text {thrust }}\left(\bar{\eta}\left(t^{n}\right)\right)+\bar{Q}_{\text {grav }}\left(\bar{\eta}\left(t^{n}\right), \zeta\left(t^{n}\right)\right),
$$

where the thrust, $\bar{Q}_{\text {thrust }}$, and gravity loads, $\bar{Q}_{\text {grav }}$, have also been included in the definition.

Equation (4) is solved together with the propagation equations that determine the position and orientation of the body-fixed reference frame. ${ }^{24}$ The equations are time-marched using an implicit, constant-acceleration Newmark integration scheme which was modified as in Géradin and Rixen ${ }^{25}$ to introduce controlled positive algorithmic damping.

\section{II.B.1. Modal Reduction of the Nonlinear System Equations}

For the efficient representation of the dynamics of the flexible aircraft, we write the perturbation equations, Eq. (4), in terms of the natural modes of the unconstrained structure at a reference condition. These vibration modes are obtained from the unforced fully-linearized version of Eq. (4), and are used to define the following transformation:

$$
\left\{\begin{array}{l}
\bar{\eta} \\
\bar{\nu}
\end{array}\right\}=\Phi\left\{\begin{array}{l}
q \\
\bar{\nu}
\end{array}\right\}
$$


where we have introduced the new variable $\bar{\nu}$ with $\dot{\bar{\nu}}=\bar{\beta}$. The vector of the projected modal coordinates is $q$ and $\Phi$ is the matrix of the corresponding mode shapes, which has been transformed to separate the rigid-body motion of the body from the elastic deformations due to the excitation of the structure. ${ }^{7} \mathrm{~A}$ required assumption for this separation is that the body-fixed frame initially coincides with the center of mass (CM) and its axes to be aligned with the principal axes of the structure. The resulting basis is found to be very convenient for the modal projection of the coupled system equations,

$$
\Phi^{\top} \mathcal{M}(\eta) \Phi\left\{\begin{array}{c}
\ddot{q} \\
\dot{\beta}
\end{array}\right\}+\Phi^{\top} \overline{\mathcal{C}}(\eta, \beta) \Phi\left\{\begin{array}{l}
\dot{q} \\
\beta
\end{array}\right\}+\Phi^{\top} \overline{\mathcal{K}}(\eta, \beta) \Phi\left\{\begin{array}{l}
q \\
0
\end{array}\right\}=\Phi^{\top} \bar{Q}_{\text {ext }}(\bar{\eta}, \dot{\bar{\eta}}, \beta, \zeta),
$$

which describes the arbitrarily large rigid-body motion of the flexible aircraft. This approach is limited to small elastic deformations which are captured using the modified vibration modes of the unconstrained structure. The modal damping and stiffness matrices remain functions of the rigid-body DoF, $\beta$. However, it is easy to see that they have, respectively, linear and quadratic dependencies with $\beta$, and that it is possible to write them in terms of third- and fourth-order tensors, ${ }^{6}$

$$
\begin{aligned}
\Phi_{i j} \overline{\mathcal{C}}_{j k} \Phi_{k l} & =c_{i l r} \beta_{r}(t), \\
\Phi_{i j} \overline{\mathcal{K}}_{j k} \Phi_{k l} & =k_{i l}^{\text {stif }}+k_{i l r s}^{\text {gyr }} \beta_{r}(t) \beta_{s}(t),
\end{aligned}
$$

where we sum over repeated indices and have identified the contributions to the modal stiffness matrix from elastic and gyroscopic forces. The tensors $c$ and $k$ are constant in time and their dimensions are $i, l=\{1, \ldots, m\}$ and $r, s=\{1, \ldots, 6\}$ for $m$ number of modes used in the expansion. These tensors are typically very sparse, and this approach generates efficient numerical solutions that keep the nonlinearities in the rigid-body DoF and all couplings with the linear structure at a low computational cost.

\section{II.C. Coupled Aeroelastic and Flight Dynamic Framework}

The presented models (and the geometrically nonlinear counterparts) have been implemented in the framework for Simulation of High Aspect Ratio Planes (SHARP) to study the behavior of flexible aircraft, including static aeroelastic analyses, trim, linear stability analyses, and fully nonlinear time-marching simulations. ${ }^{4,6,20,21,26}$ In this section we will focus on coupling the linearized models which form the basis for subsequent model reduction.

\section{II.C.1. Monolithic Framework for Linearized Flexible-Aircraft Dynamics}

Murua et al. ${ }^{4}$ demonstrated that the linearized aerodynamic formulation, Eq. (1), enables rigid-body motions and elastic deformations (both small) to be incorporated in a unified monolithic framework. This provides a powerful formulation to determine the aircraft dynamic stability and it can be very useful for efficient control design and optimization. As the linear UVLM is written in discrete time, temporal discretization of the flexible-body system, linear form of Eq. (4), is also required before the fluid/structure coupling. A standard Newmark- $\beta$ discretization is used, which can also include algorithmic damping in the structural dynamics response. After introducing the modal transformation of Eq. (6), this leads to the following aeroelastic state-space system ${ }^{20}$

$$
\begin{aligned}
\Delta x^{n+1} & =A_{A S} \Delta x^{n}+B_{A S} \Delta u_{A S}^{n} \\
\Delta y_{A S}^{n} & =C_{A S} \Delta x^{n},
\end{aligned}
$$

where the state vector that completely determines the linear system is

$$
x=\left[x_{A}^{T} \mid x_{S}^{T}\right]^{T}=\left[\begin{array}{lllll}
\Gamma_{b}^{T} & \Gamma_{w}^{T} \dot{\Gamma}_{b}^{T} \mid q^{T} \dot{q}^{T} & \beta^{T} & \Theta^{T}
\end{array}\right]^{T},
$$

and $u_{A S}$ represents the (aerodynamic) inputs to the system to account for gusts and control inputs in a (closed-loop) time-marching solution. The subscript $A S$ is introduced here to separate the aeroelastic system variables from the linear equations of the UVLM in Eq. (1). Depending on the nature of the problem, the system output vector $y_{A S}$ can include aerodynamic loads, elastic deformations, aircraft position, attitude, etc. From the homogeneous form of Eq. (9) one also obtains a discrete-time generalized eigenvalue problem to determine the dynamic stability of the vehicle which includes aeroelastic and flight dynamic modes. 


\section{II.C.2. Dynamic Response of Maneuvering Flexible Aircraft}

The linear equations above may be enough for stability augmentation in forward flight, but it cannot describe arbitrary vehicle maneuvers. In this section we present the equations of motion of flexible aircraft undergoing large maneuvers, e.g. due to gusts or control surface inputs. The resulting system is nonlinear because of gyroscopic forces dominating the flight dynamics, however, under the assumption of small elastic deformations of lifting surfaces (during the transient dynamics, the reference condition may still have large displacements) we can assume that the coupled aerodynamics are linear. Unlike aeroelastic approaches based on frequency-domain aerodynamics, the flexible-aircraft dynamics model in this work is developed by tightly coupling the modal form of the flexible-body equations, Eq. (7), with the linear unsteady vortex lattice model, Eq. (1).

The resulting description is based on the vibration modes of the unconstrained flexible body, $\Phi$, to reduce (a) the size of the nonlinear flexible-body dynamics problem, and (b) the number of inputs and outputs of the aerodynamic system, Eq. (1). The resulting aerodynamic system can be projected such that

$$
\begin{aligned}
\Delta \Gamma^{n+1} & =A \Delta \Gamma^{n}+B_{S} \Phi \Delta u_{\Phi}^{n}+B_{A} \Delta u_{A}^{n} \\
\Delta y_{A}^{n} & =\Phi^{\top} C \Delta \Gamma^{n}+\Phi^{\top} D_{S} \Phi \Delta u_{\Phi}{ }^{n}+\Phi^{\top} D_{A} \Delta u_{A}{ }^{n} .
\end{aligned}
$$

where the inputs to the aerodynamic model are now the generalized coordinates of the flexible-body equations, i.e. $\Delta u_{\Phi}=\left[q^{\top} \beta^{\top}\right]^{\top}$, while the aerodynamic inputs, $u_{A}$, still account for gust induced velocities and the deployment of control surfaces. The output vector $y_{A}$ contains the unsteady loads due to the excitation of a certain vibration mode to obtain the generalized forces. Other applied loads, such as thrust and gravity, are assembled in the flexible-body dynamics subsystem, as shown in Eq. (5), and finally complete the generalized force vector $\Phi^{\top} \bar{Q}_{\text {ext }}$ in Eq. (7). The tight coupling between the aerodynamic and the flexible-body dynamic models is illustrated in Fig. 1.

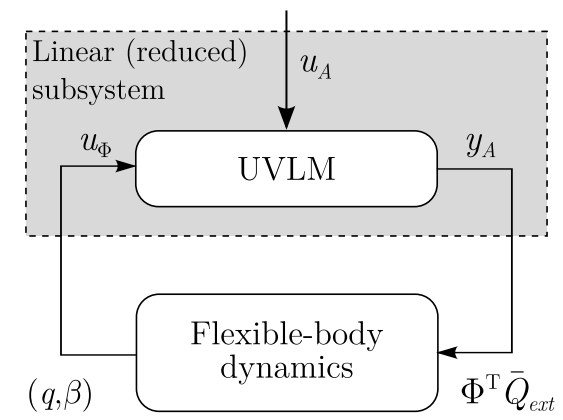

Figure 1: Time-marching solution for maneuvering flexible aircraft.

Even though the global coupled system equations are still nonlinear, we have isolated the linear aerodynamic subsystem, which is to be solved at every subiteration in the solution process. Similar to the monolithic system, the aerodynamic system requires a large number of states to transfer the information from a small number of inputs (structural mode shapes) to a small number of outputs (generalized forces). Model reduction of the linear system will be the focus of the next section, where we can control the number of system inputs and outputs by selecting only a few vibration modes for the structural problem.

Note that this approach does not require transformation to frequency domain to obtain the generalized forces, but the linearization of the unsteady aerodynamics is limited to a specific aeroelastic reference condition with the dynamic pressure as a parameter. This requires interpolation of the aerodynamic (reduced-order) model between different parameter values, such as flight speed or altitude, to enlarge the flight envelope of very flexible aircraft configurations. ${ }^{27}$ However, if the linearization is done around the undeformed reference, as in DLM solutions, it is easy to show that the aerodynamic loads in the resulting linear system, Eq. (11), are proportional to the forward-flight dynamic pressure. This would generate reduced-order aerodynamic models which are independent of the flight vehicle flight conditions, but this also constraints the admissible wing kinematics and will not be further investigated in this paper. 


\section{Reduced-Order Coupled Aeroelasticity and Flight Dynamics}

The size of the linear systems presented in Eqs. (11) and (9) is dominated by the number of vortex panels used in the discretization of lifting surfaces and the wake of the aircraft. Wake panels are needed to capture the unsteady effects, but they are also an excellent target for model reduction. A direct approach to reduce the size of the aeroelastic system is modal projection (and truncation) of the system states on a few generalized coordinates. ${ }^{8}$ However, as we discussed in the introduction, this approach may require many states in the reduced-order model to capture the internal dynamics of the flow. ${ }^{14,18} \mathrm{~A}$ more efficient basis function can be found by balancing the system so that the input-output mapping drives the reduction process. ${ }^{14-16}$ Such balancing-based model reduction techniques achieve optimal reductions for stable systems in continuous time, but application to integrated aeroelastic systems, such as the one in Eq. (9), requires a different reduction approach to handle unstable poles. Such instabilities are not only due to the physics of the problem (e.g. flutter), but also due to the numerical inaccuracy at very high frequencies. Although the latter results in marginally-stable, high-frequency aeroelastic modes which contribute little to the system response, they are preserved in the standard balanced realization method. ${ }^{16}$ A more robust approach is shown in Figure 2, and would start with truncation of high-frequency (unstable) modes with a subsequent model reduction of the remaining, possibly unstable, aeroelastic system. This will be described next.

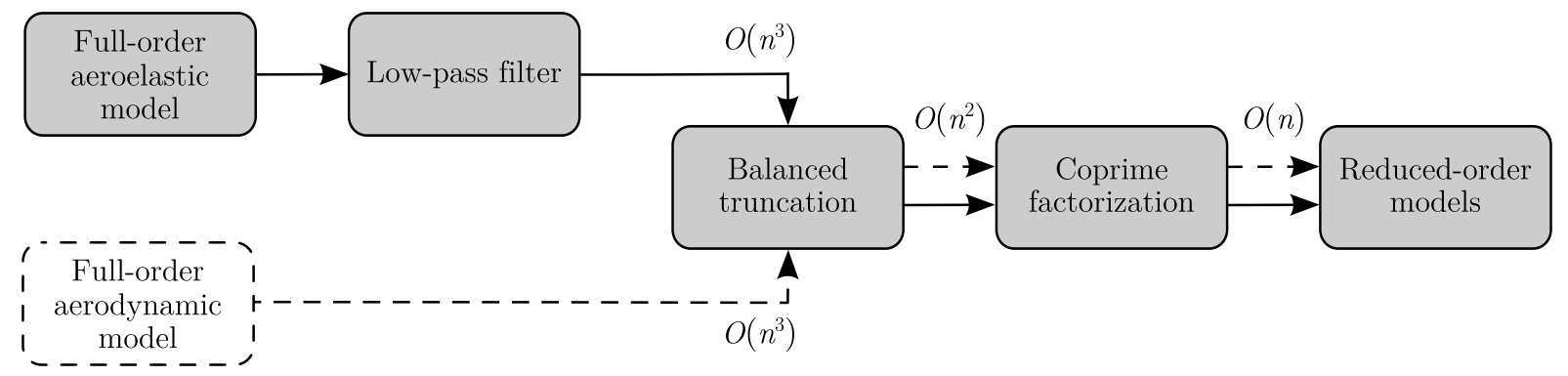

Figure 2: Approach for model reduction of the aeroelastic and aerodynamic systems with the model orders, $n$, shown as an indication for problem sizes discussed in this work.

In a first step, the aeroelastic system is filtered to either stabilize or remove high-frequency or highlydamped modes which have negligible contribution to the system dynamics. As mentioned above, this step enables a more efficient balanced truncation of the system without detrimental effects on accuracy. Two methods have been explored in this work. The modification of the Newmark integration scheme in the discretization of the flexible-body dynamics EoM to include positive algorithmic damping ${ }^{25}$ provides a very effective way to stabilize high-frequency aeroelastic modes without any significant effect on the dominant flight dynamic modes. As a second approach, Schur decomposition can also be applied to the full-order system to remove high-frequency or highly-damped modes. The Nyquist rate can be used to obtain a threshold frequency for truncation of high-frequency zero or unstable poles. A conventional modal truncation approach $^{28}$ was found to perform poorly, as the modal basis for projection of the large aeroelastic system often becomes rank deficient at working precision. Transforming the system matrix to its Schur form on the contrary, has stabilizing numerical properties. ${ }^{29}$ However, for the numerical results presented in this work, small algorithmic damping in the temporal discretization was found to be sufficient to ensure robustness of the model reduction and the Schur decomposition was not pursued.

Subsequent balanced truncation significantly reduces the system size using a priori error bounds as an indication for the truncation order. Even further reduction with comparable system mismatch is achieved through subsequent truncation of the system normalized coprime factors. ${ }^{30}$ This approach was originally developed for unstable plants, but was found to be more effective in the reduction of the aeroelastic system with poles close to the stability boundary than the standard balanced truncation technique. The coprime factorization approach, however, is much more computationally expensive and a combination of initial balanced truncation to the order $\mathcal{O}\left(n^{2}\right)$ followed by coprime factorization of the truncated system, as indicated in Figure 2, was found to be a better compromise for aeroelastic applications.

The aerodynamic subsystem for the analysis of maneuvering aircraft on the other hand, is highly damped and the integrated approach of initial balanced realization followed by truncation of the normalized coprime factors is proposed without the initial low-pass frequency filter, as described in Figure 2. Each reduction 
technique is discussed briefly in the following subsections for completeness.

\section{III.A. Balanced Realization and Truncation}

By balancing the states of the linear systems, the transmission path between the system inputs and outputs is captured in an optimal way. This approach was first discussed by Moore, ${ }^{15}$ while a detailed description is provided by Glover. ${ }^{16}$ Information about the past input and future output energy, expressed in terms of the system controllability and observability Gramians, is used to identify a linear similarity transformation $T$ that balances the stable system $\{A, B, C, D\}$, such that each balanced state is equally controllable and observable. The balanced system can then be written in terms of the transformed state coordinates $\hat{x}=T^{-1} x$ as

$$
\begin{aligned}
\Delta \hat{x}^{n+1} & =T^{-1} A T \Delta \hat{x}^{n}+T^{-1} B \Delta u^{n} \\
\Delta y^{n} & =C T \Delta \hat{x}^{n}+D \Delta u^{n} .
\end{aligned}
$$

Note that in this section we present the balancing method for both linear systems, Eqs. (11) and (9), and the subscripts $A$ and $A S$ have been dropped in this discussion. Expressions for the discrete-time counter part exist, but we will follow the standard implementation of these methods, which involves a bilinear transformation to continuous time prior to any model reduction. ${ }^{31}$

The similarity transformation $T$ is obtained using the controllability and observability Gramians, $W_{c}$ and $W_{o}$, respectively, which are given for stable LTI systems by the algebraic Lyapunov equations,

$$
\begin{aligned}
& A W_{c}-W_{c} A^{\top}+B B^{\top}=0, \\
& A^{\top} W_{o}-W_{o} A+C^{\top} C=0 .
\end{aligned}
$$

where the controllability Gramian, $W_{c}$, is correlated inversely to the input energy required by an actuator to reach a certain state and the observability Gramian, $W_{o}$, on the contrary, is related directly to the energy required by a sensor to observe an initial state. ${ }^{32}$

The square root of the eigenvalues of the Gramian product, $W_{c} W_{o}$, are the Hankel singular values (HSV) of the system denoted as $\sigma_{1} \geq \sigma_{2} \geq \cdots \geq \sigma_{n}>0 .{ }^{33}$ The HSV are invariant to the transformation and the Gramians of the balanced system, $\hat{W}_{c}=T^{-1} W_{c} T^{-\top}$ and $\hat{W}_{o}=T^{\top} W_{o} T$, are chosen such that they are equal and diagonal, $\hat{W}_{c}=\hat{W}_{o}=\Sigma=\operatorname{diag}\left(\sigma_{1}, \ldots, \sigma_{n}\right)$. Hence the states with the largest HSV are the ones most involved in the energy transfer between the system inputs and outputs and the least controllable/observable states can be truncated.

The procedure to obtain the balancing transformation, $T$, is outlined in Algorithm 1, which follows closely the Square Root Method presented by Laub et al. ${ }^{34}$ The Choleski factors $P$ and $Q$ of the Gramians in the first step are computed directly from the state-space representation $\{A, B, C, D\}$, using the method of Hammerling, ${ }^{35}$ without the need to evaluate $W_{c}$ and $W_{o}$ which is costly and ill-conditioned. Reduction of the balanced system, obtained from Eq. (12), can be achieved finally by truncating the corresponding rows and columns of the matrix $T$ according to the HSV of each balanced state, $\sigma_{i}$.

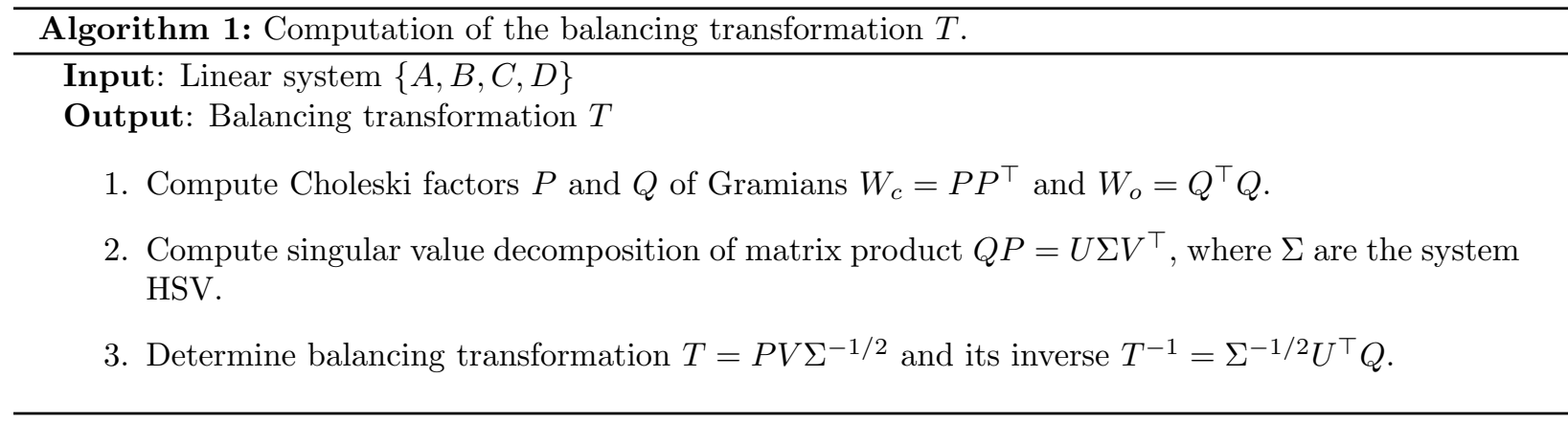

\section{III.B. Normalized Coprime Fractional Balancing for Unstable Systems}

The balanced truncation method is limited to stable systems, which is a necessary assumption to compute the controllability and observability Gramians using the Lyapunov equations, Eq. (13). We also found that it behaves poorly for marginally-stable systems, which can hinder its application to aeroelastic systems 
with poles close to the stability boundary. Enns ${ }^{36}$ has proposed a solution to tackle this issue whereby the unstable system $\{A, B, C, D\}$ is divided into a stable and an unstable part and only the stable part is reduced through balanced truncation. Since this approach retains all unstable poles without reduction, it can yield unnecessarily-large reduced-order systems.

Alternatively, Meyer ${ }^{30}$ has presented a balancing method for unstable strictly proper systems based on coprime factorization. The idea is to represent the minimal realization of the full system $G(s)=$ $C(s I-A)^{-1} B$ in terms of its stable normalized right coprime factors (NRCF), $N_{r}(s)$ and $M_{r}(s)$, such that $G(s)=N_{r}(s) M_{r}^{-1}(s) .{ }^{37}$ To ensure uniqueness of the solution we also need to enforce $N_{r}^{\top}(-s) N_{r}(s)+$ $M_{r}^{\top}(-s) M_{r}(s)=I, \forall s$. Note that we have switched to continuous time to obtain the system NRCF.

Following the approach by Meyer and Franklin, ${ }^{38}$ we can introduce state feedback to stabilize the system. This results in a modified system, $\bar{A}=A+B K$, from which we can now obtain the state-space realizations for the stable NRCF as

$$
\begin{aligned}
& N_{r}(s)=C(s I-\bar{A})^{-1} B \\
& M_{r}(s)=I+K(s I-\bar{A})^{-1} B
\end{aligned}
$$

where the state feedback gain in continuous time can be computed as $K=-B^{\top} P$ with $P$ being the solution of the algebraic Riccati equation ${ }^{39}$

$$
P A+A^{\top} P-P B B^{\top} P+C^{\top} C=0
$$

The state-space realization of the resulting pair of stable transfer functions $\left(N_{r}^{\top}(s), M_{r}^{\top}(s)\right)^{\top}$ is given as

$$
\left\{\bar{A}, B,\left(\begin{array}{l}
C \\
K
\end{array}\right),\left(\begin{array}{l}
0 \\
I
\end{array}\right)\right\},
$$

which can be balanced and truncated following the procedure outlined in Section III.A to obtain the stable reduced-order model $\left\{\bar{A}_{11}, B_{1},\left(\begin{array}{c}C_{1} \\ K_{1}\end{array}\right),\left(\begin{array}{l}0 \\ I\end{array}\right)\right\}$. Reversal of the state feedback and output augmentation finally leads to the reduced-order unstable model $\left\{A_{11}, B_{1}, C_{1}\right\}$ of the plant $G(s) .{ }^{30}$ Following Vidyasagar, ${ }^{40}$ the normalized coprime factorization method was also finally extended to balance the non-strictly proper aerodynamic system in Eq. (11).

\section{Numerical Studies}

This section will demonstrate the model reduction approaches on several aeroelastic problems of increasing complexity. The methods were implemented in the multidisciplinary environment for the Simulation of HighAspect-Ratio Planes (SHARP) developed by the authors and co-workers. ${ }^{6,7,21,26}$ The Goland wing problem will first serve to demonstrate the reduced-order modeling approach on an unstable linear aeroelastic system, given by the monolithic framework presented in Eq. (9) (neglecting the rigid-body DoF), with the aim to synthesize a robust flutter suppression controller. In the second subsection the focus will be on model reduction of a maneuvering high-altitude, long-endurance (HALE) aircraft subject to control surface inputs.

\section{IV.A. Model Reduction for the Goland Wing Problem}

The Goland wing is a simple numerical benchmark that has been studied extensively in literature ${ }^{21,41-43}$ and it will be used in this study to explore the balanced truncation method for the linear aeroelastic system, Eq. (9). It is a stiff cantilever for which Table 2 summarizes the relevant properties. ${ }^{21}$

To determine an appropriate spatial discretization in the full-order model, a convergence study is conducted to obtain the aeroelastic flutter boundary of the wing, which is obtained from the homogeneous form of Eq. (9). See Murua et al. ${ }^{21}$ for a detailed description of the flutter analysis in SHARP. The convergence in this coupled problem is dictated by the UVLM discretization and results in $M=16$ chordwise and $N=26$ spanwise panels. To ensure mesh consistency, varying the number of chordwise bound panels also affects the time step of the discrete-time problem and, hence, the number of panels used to discretize the wake, where a wake length of 15 chord lengths was kept in this study as determine by another convergence study. This results in over 4500 states to solve the flutter problem accurately with a tolerance of $\pm 0.5 \mathrm{~m} / \mathrm{s}$. With this, the flutter speed is $V_{f}=169 \mathrm{~m} / \mathrm{s}$, and frequency, $\omega_{f}=70 \mathrm{rad} / \mathrm{s}$, which is in excellent agreement with the 
Table 2: Goland wing properties. ${ }^{21}$

\begin{tabular}{lrlr}
\hline \hline Chord, $c$ & $1.8288 \mathrm{~m}$ & Mass per unit length & $35.71 \mathrm{~kg} / \mathrm{m}$ \\
Semi-span & $6.096 \mathrm{~m}$ & Moment of inertia & $8.64 \mathrm{~kg} \cdot \mathrm{m}$ \\
Elastic axis (from l.e.) & $0.33 \cdot c$ & Torsional stiffness & $0.99 \times 10^{6} \mathrm{~N} \cdot \mathrm{m}^{2}$ \\
Center of gravity (from l.e.) & $0.43 \cdot c$ & Bending stiffness & $9.77 \times 10^{6} \mathrm{~N} \cdot \mathrm{m}^{2}$ \\
\hline \hline
\end{tabular}

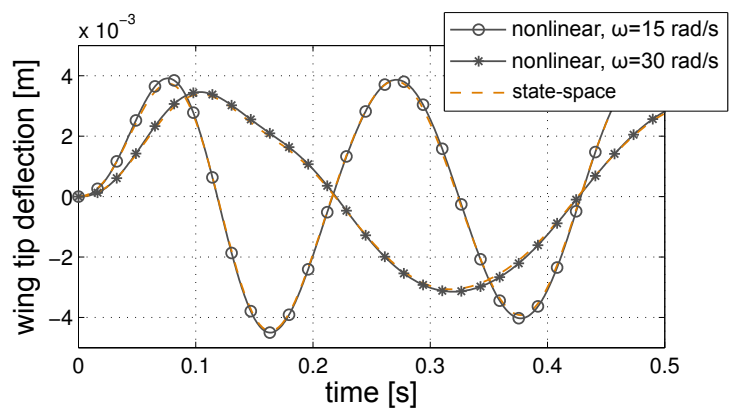

(a) Forced vibration for $V=140 \mathrm{~m} / \mathrm{s}$ and frequencies, $\omega$

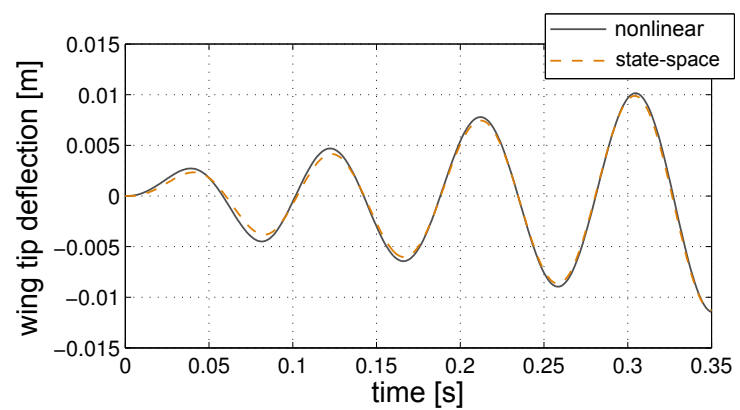

(b) Free vibration for $V=180 \mathrm{~m} / \mathrm{s}$

Figure 3: Open-loop response of the Goland wing subject to sinusoidal aileron input with 1 deg amplitude at different free-stream velocities, $V$.

values reported in the literature. ${ }^{21,43}$ The flutter boundary, however, is sensitive to small variations in speed and a smaller number of panels may be needed at lower, stable free-stream velocities.

The converged state-space aeroelastic system with $16 \times 26$ bound panels ( 4550 aeroelastic states) is then verified against a nonlinear time-marching solution at two different free-stream velocities of $V=140 \mathrm{~m} / \mathrm{s}$ $\left(0.83 V_{f}\right)$ and $V=180 \mathrm{~m} / \mathrm{s}\left(1.07 V_{f}\right)$, respectively. The reference solution is obtained using the geometrically nonlinear aeroelastic solver in SHARP with a nonlinear implementation of the UVLM ${ }^{21,26}$ based on the description by Katz and Plotkin. ${ }^{44}$ The single input to the linear system operates an aileron of $25 \%$ chord length fixed to the last quarter of the wing and the system output is the vertical tip deflection. The open-loop response to a sinusoidal input of 1 deg amplitude in Figure 3(a) shows good comparison between the two solution methods below flutter speed over a range of excitation frequencies, $\omega$. The (free) flutter response, as shown in Figure 3(b) for $V=180 \mathrm{~m} / \mathrm{s}\left(1.07 V_{f}\right)$, is obtained following an initial sinusoidal excitation of the aileron for one period with $\omega=70 \mathrm{rad} / \mathrm{s}$ and again $1 \mathrm{deg}$ amplitude. Both solutions predict the exponential growth of the wing tip deflection at small amplitudes. Hence, if the size of the state-space aeroelastic model is reduced significantly and the resulting vibrations remain small, such a system can be used for robust flutter suppression using $\mathcal{H}_{\infty}$ control $^{45}$ and will provide the motivation here for model reduction of the aeroelastic system, as outlined in Section III.

\section{IV.A.1. Model Reduction Using a Balanced Coprime Factorization Approach}

A number of numerical issues need to be addressed before balancing methods can be applied to the aeroelastic system. Figure 4(a) demonstrates the effect of coupling the highly-damped aerodynamic modes with the structural system, which can lead to lightly-damped (or even unstable) poles at the high frequency range above 50 times the flutter frequency of the coupled aeroelastic system. These unnecessary modes can hamper the model reduction, which is overcome by introducing algorithmic damping to the discretization of the structural dynamics problem using an implicit Newmark- $\beta$ integration scheme. ${ }^{25}$ As a result, the undesired high-frequency modes are shifted away from the stability boundary while the dominant modes are preserved, as shown in Figure 4(b). Algorithmic damping of $c_{n m}=0.005$ will be applied in this study. Note that the imaginary part of the eigenvalues in Figure 4 has been normalized with $c /(2 V)$ to present the modes in terms of their reduced frequencies while no normalization was applied to damping.

Following the model reduction approach outlined in Figure 2, the (unstable) full-order aeroelastic system above flutter speed $\left(1.07 V_{f}\right)$ is firstly reduced to 500 states through balanced truncation of the (unstable) 


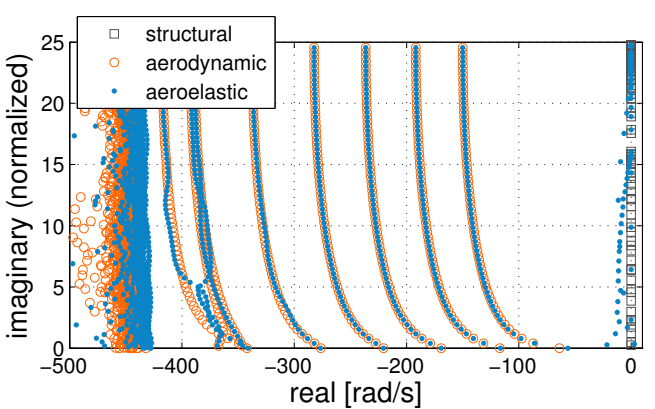

(a) Coupling effects without numerical damping

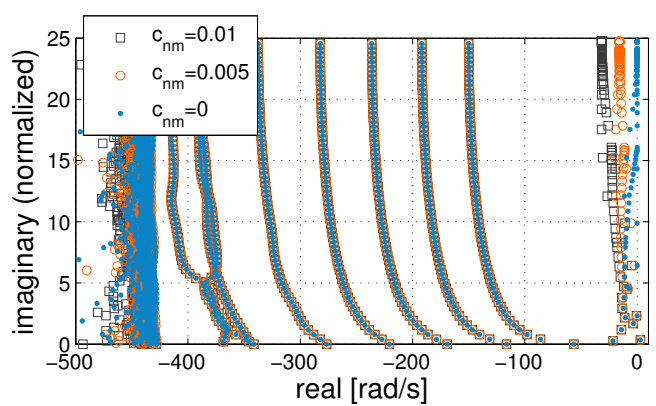

(b) Effect of numerical damping, $c_{n m}$, on aeroelastic modes

Figure 4: Root locus of the Goland wing at free-stream velocity $V=180 \mathrm{~m} / \mathrm{s}\left(1.07 V_{f}\right)$ showing the modes of the structural (Eq. (6)), aerodynamic (Eq. (1)), and the coupled aeroelastic (Eq. (9)) systems. Eigenvalues have been converted to continuous time and imaginary part normalized with $c /(2 \mathrm{~V})$.

plant which dramatically improves numerical efficiency of the subsequent reduction using the normalized coprime factorization method. Figure 5 shows the model reduction in terms of the $\nu$-gap metric which provides a measure of similarity of the input-output mapping between the original and the reduced-order system (mapping is identical for zero and completely dissimilar for one). ${ }^{46}$ For completeness we also included the $\nu$-gap metric of a simple balanced realization without coprime factorization, which shows that the proposed balanced coprime method can achieve better reduced-order model fidelities of the unstable system compared to the standard balanced truncation method. The relative Hankel singular values of the balanced system are also superimposed in Figure 5 . The HSV indicate the contribution of each balanced state to the system input-output mapping. The relative values are normalized in this example with respect to the third state of the balanced system, as the first two states have infinite HSV corresponding to the unstable complex conjugate pair of the flutter mode. As expected, the HSV of the balanced system are closely correlated to the $\nu$-gap metric of the reduced-order models and provide an error bound of the model reduction before the truncation.

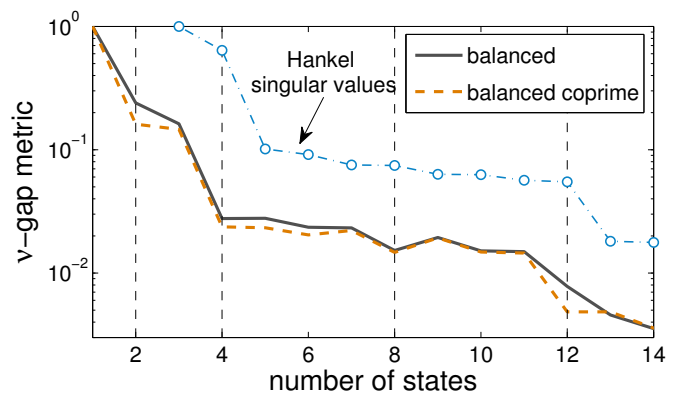

Figure 5: The $\nu$-gap metric between the full-order model and the reduced-order systems obtained using the standard balanced truncation and the balanced coprime factorization method. Superimposed are the Hankel singular values of the balanced system normalized with the third state.

Based on the HSV of the balanced system, we chose to compare four different reduced-order models with 2, 4, 8, and 12 states, as highlighted in Figure 5, where the first two states correspond to the unstable flutter mode. Figure 6 compares the Bode response of the selected reduced-order systems with the full-order model response over a range of reduced frequencies, $k=\omega c /(2 \mathrm{~V})$. It is clear from the results that only two balanced states are needed to capture the coupling of the complex 3D unsteady flow characteristics and the structural response of the Goland wing around the flutter point. More states are required to also capture the higher-frequency response, but as little as 12 states are sufficient to predict the response of the fullorder system with 4550 states even at high reduced frequencies of $k=3$. These results show the potential of reduced-order modeling using balancing methods, even for relatively large, unstable aeroelastic systems, leading to substantial reductions of three to four orders of magnitude which is a requisite for robust control synthesis using higher-fidelity models. ${ }^{47,48}$ 

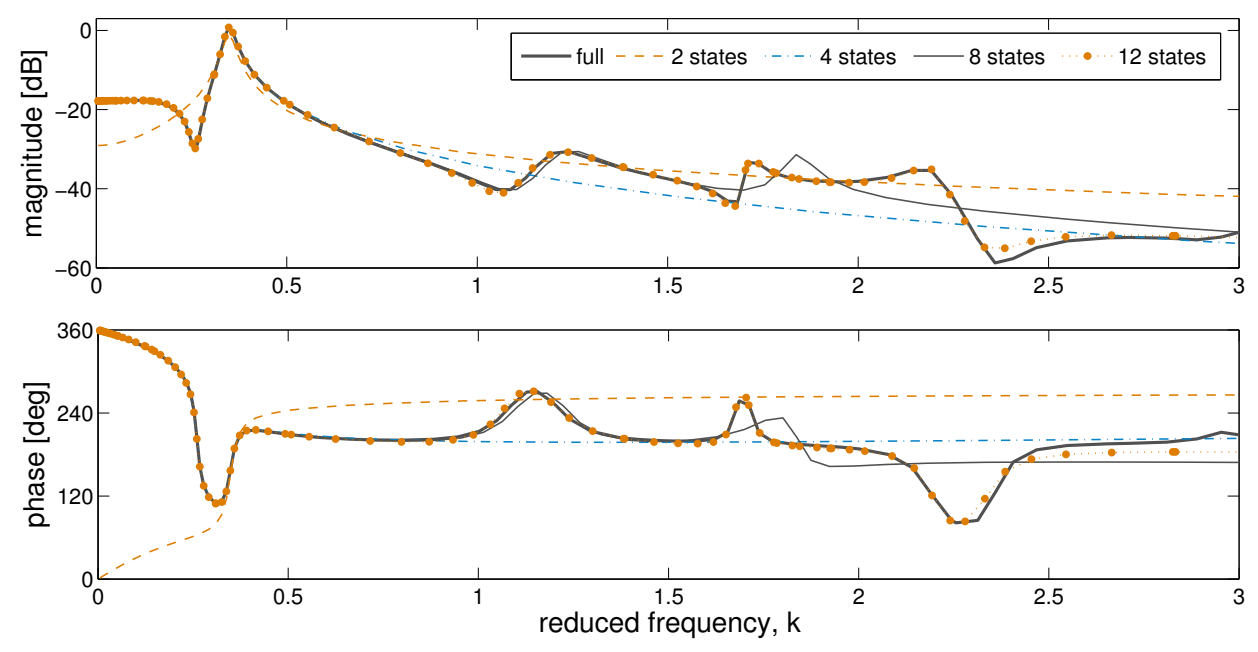

Figure 6: Comparison of different reduced-order balanced system responses against the full-order model for a range of reduced frequencies, $k=\omega c /(2 V)$, at free-stream velocity $V=180 \mathrm{~m} / \mathrm{s}\left(1.07 V_{f}\right)$.

\section{IV.A.2. Robust Control Synthesis for Flutter Suppression}

Next, we will exercise the reduced-order system to compute a robust $\mathcal{H}_{\infty}$ controller to alleviate vibrations, as demonstrated in Cook et al. ${ }^{47}$ for gust alleviation of very flexible aircraft. In this work we will demonstrate the controller for flutter suppression of the Goland wing at a free-stream velocity $V=180 \mathrm{~m} / \mathrm{s}\left(1.07 V_{f}\right)$. Even though pole placement would be the natural choice to directly stabilize the flutter mode, the flutter suppression problem provides a simple environment to demonstrate the $\mathcal{H}_{\infty}$ control synthesis based on a reduced-order model, which can be applied to gust alleviation in future work.

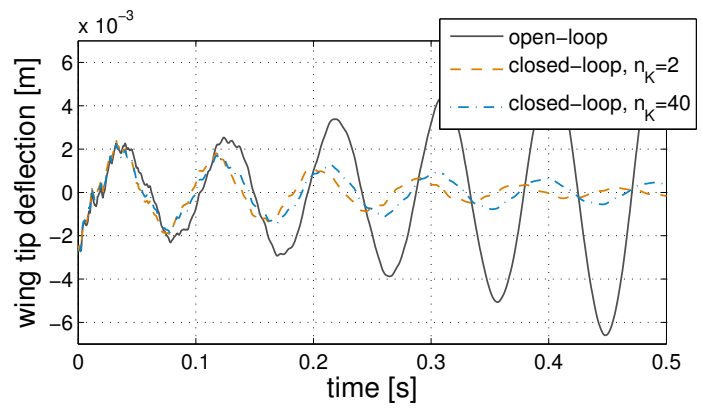

(a) State-space open- and closed-loop impulse response

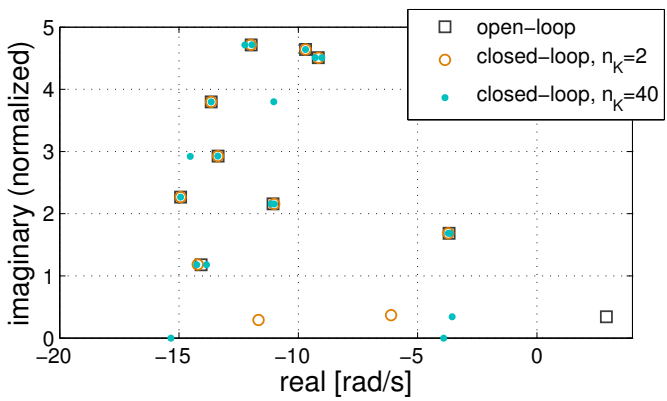

(b) Root locus of open- and closed-loop systems

Figure 7: Demonstration of flutter suppression for the Goland wing at free-stream velocity $V=180 \mathrm{~m} / \mathrm{s}$ $\left(1.07 V_{f}\right)$ for varying number of $\mathcal{H}_{\infty}$ controller states, $n_{K}$. Eigenvalues have been converted to continuous time and imaginary part normalized with $c /(2 \mathrm{~V})$.

Following $\mathrm{Ng}$ et al. ${ }^{48}$ the aeroelastic system is expanded to account for a disturbance of the aileron in the form of an initial $0.1 \mathrm{rad}$ step input. The objective of the controller is to minimize the resulting vertical wing tip deflection using the aileron, where the disturbance and control inputs to the linear aeroelastic system are treated as separate control surfaces. The MATLAB Robust Control Toolbox ${ }^{31}$ is used to obtain the controller for the given disturbance using the weights $W_{1}=2$ and $W_{2}=3$ on the system states and inputs, respectively, while no measurement noise was assumed. To demonstrate the application of the model reduction, the controller is synthesized based on the reduced-order system with $n_{K}$ states and is then applied to the full-order system to stabilize the response. Figure 7 (a) compares the state-space open-loop and closedloop responses, which suggests that two controller states are sufficient to suppress flutter and that using more states in the control synthesis can deteriorate the controller performance.

This is also reflected in the root locus of the closed-loop system shown in Figure 7 (b) for the controller states $n_{K}=2$ and 40, which correspond to the extreme cases considered in this study. The controller 
effectiveness varies with increasing number of controller states, $n_{K}$, but it can be seen from Figure 7 (b) that the poles of the closed-loop systems converge away from the flutter boundary. Hence, this simple example demonstrates that a very low-order, reduced system is sufficient to capture most features of the unsteady flow and the resulting structural vibrations which can be used for very efficient synthesis of a robust flutter suppression controller.

\section{IV.B. Reduced-Order Modeling of a Flexible HALE Configuration}

In this section we will demonstrate the reduced-order modeling approach to compute the open-loop response of a flexible maneuvering aircraft. A representative HALE configuration, shown in Figure 8, was introduced in the previous study ${ }^{7}$ with a detailed description of the aircraft and its material properties which are repeated in Table 3 for completeness. The aircraft is similar to those by Patil et al. ${ }^{49}$ and Murua et al. ${ }^{4}$ but it includes winglets to increase resistance against spiral divergence. The vehicle is powered by two massless propellers, which are modeled as point forces rigidly linked to the wing. The mass per unit length of the fuselage is the same as that of the horizontal and vertical tail planes, and thus the total mass of this aircraft, including payload and structural mass, is $75.4 \mathrm{~kg}$.

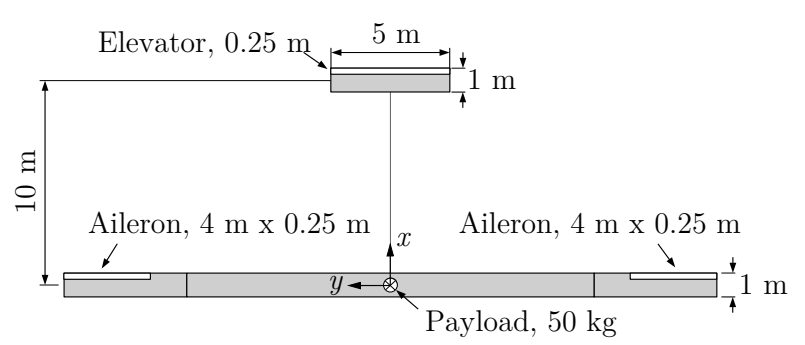

(a) Top view

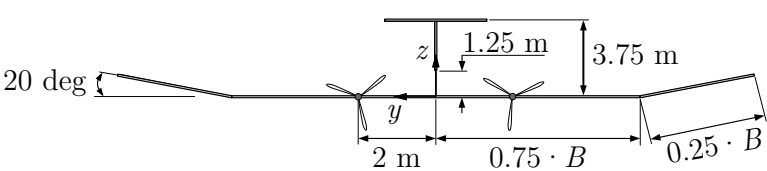

(b) Front view

Figure 8: Undeformed HALE aircraft geometry (not to scale). ${ }^{7}$

Table 3: HALE aircraft properties. ${ }^{7}$

\begin{tabular}{lll}
\hline \hline & Main wing & Tail plane \\
\hline Chord, $c$ & $1 \mathrm{~m}$ & $0.5 \mathrm{~m}$ \\
Semi-span, $B$ & $16 \mathrm{~m}$ & $2.5 \mathrm{~m}$ \\
Elastic axis (from l.e.) & $0.5 \mathrm{~m}$ & $0.25 \mathrm{~m}$ \\
Center of gravity (from l.e.) & $0.5 \mathrm{~m}$ & $0.25 \mathrm{~m}$ \\
Mass per unit length & $0.75 \mathrm{~kg} / \mathrm{m}$ & $0.08 \mathrm{~kg} / \mathrm{m}$ \\
Moment of inertia & $0.1 \mathrm{~kg} \cdot \mathrm{m}$ & $0.01 \mathrm{~kg} \cdot \mathrm{m}$ \\
Torsional stiffness & $1 \sigma \times 10^{4} \mathrm{~N} \cdot \mathrm{m}^{2}$ & $\infty$ \\
Bending stiffness & $2 \sigma \times 10^{4} \mathrm{~N} \cdot \mathrm{m}^{2}$ & $\infty$ \\
In-plane bending stiffness & $4 \sigma \times 10^{6} \mathrm{~N} \cdot \mathrm{m}^{2}$ & $\infty$ \\
\hline \hline
\end{tabular}

The parameter $\sigma$ will be used to linearly vary the stiffness properties of the main wing (decreasing $\sigma$ results in a more flexible configurations), whereas fuselage and T-tail are assumed to be rigid. The vehicle is trimmed for each $\sigma$ at a free-stream velocity of $V_{\infty}=30 \mathrm{~m} / \mathrm{s}$ and an altitude of $20 \mathrm{~km}$ (air density $\rho=0.0889$ $\mathrm{kg} / \mathrm{m}^{3}$ ) to obtain the geometrically nonlinear, deformed configuration at steady level flight. The parameter $\sigma=1$ corresponds to a very flexible aircraft configuration with wing tip deflections of $42 \%$ compared to the semispan of the main wing, $B=16 \mathrm{~m}$. The deformation for $\sigma=1000$ is $0.03 \%$. The trim inputs for each aircraft configuration are given in detail in Hesse et al. ${ }^{7}$ Table 4 shows the (flexible) flight dynamics modes and note in particular the lightly-damped phugoid and spiral modes. The effect of geometric nonlinearity on the wing deformations shown in terms of the tip deflections at trimmed flight is also demonstrated in the table. 
Table 4: Stability characteristics of the HALE aircraft and normalized wing tip deflection at trimmed flight for varying stiffness parameter $\sigma$.

\begin{tabular}{cccccc}
\hline \hline$\sigma$ & Trim deflection & Phugoid & Spiral & Dutch roll & Short period \\
\hline 2 & $0.360 \cdot B \cdot \sigma^{-1}$ & $-0.001 \pm 0.30 i$ & -0.087 & $-0.31 \pm 1.27 i$ & $-2.34 \pm 1.62 i$ \\
5 & $0.336 \cdot B \cdot \sigma^{-1}$ & $-0.007 \pm 0.29 i$ & -0.041 & $-0.35 \pm 1.29 i$ & $-3.97 \pm 2.56 i$ \\
10 & $0.330 \cdot B \cdot \sigma^{-1}$ & $-0.009 \pm 0.28 i$ & -0.028 & $-0.36 \pm 1.30 i$ & $-4.79 \pm 1.88 i$ \\
1000 & $0.324 \cdot B \cdot \sigma^{-1}$ & $-0.011 \pm 0.27 i$ & -0.016 & $-0.37 \pm 1.30 i$ & $-5.35 \pm 0.94 i$ \\
\hline \hline
\end{tabular}

\section{IV.B.1. Eigenvalue analysis}

The dynamic stability characteristics of the deformed HALE configuration are obtained for each trim solution from the eigensolution of the homogeneous form of Eq. (9) which integrates the aircraft aeroelastic and flight dynamic response in a monolithic framework. The total system size of the converged problem -8 bound panels per chordwise meter and 2 panels per spanwise meter of lifting surfaces - is 6171 states which includes 5094 aerodynamic states, 1068 structural states, and 9 rigid-body states. Figure 9 presents the root locus of the linear flexible-aircraft dynamics system as a function of the wing stiffness, $\sigma$. As the aeroelastic system is derived in discrete time, we have converted the discrete-time eigenvalues to continuous time for an easier interpretation and the imaginary part of the eigenvalues has been normalized again with $c /(2 \mathrm{~V})$ to present the modes in terms of reduced frequency. Figure 9(a) only shows the roots near the origin and the highlydamped aerodynamic modes as well as the lightly-damped aeroelastic and flight dynamic modes have been identified by their mode shapes.

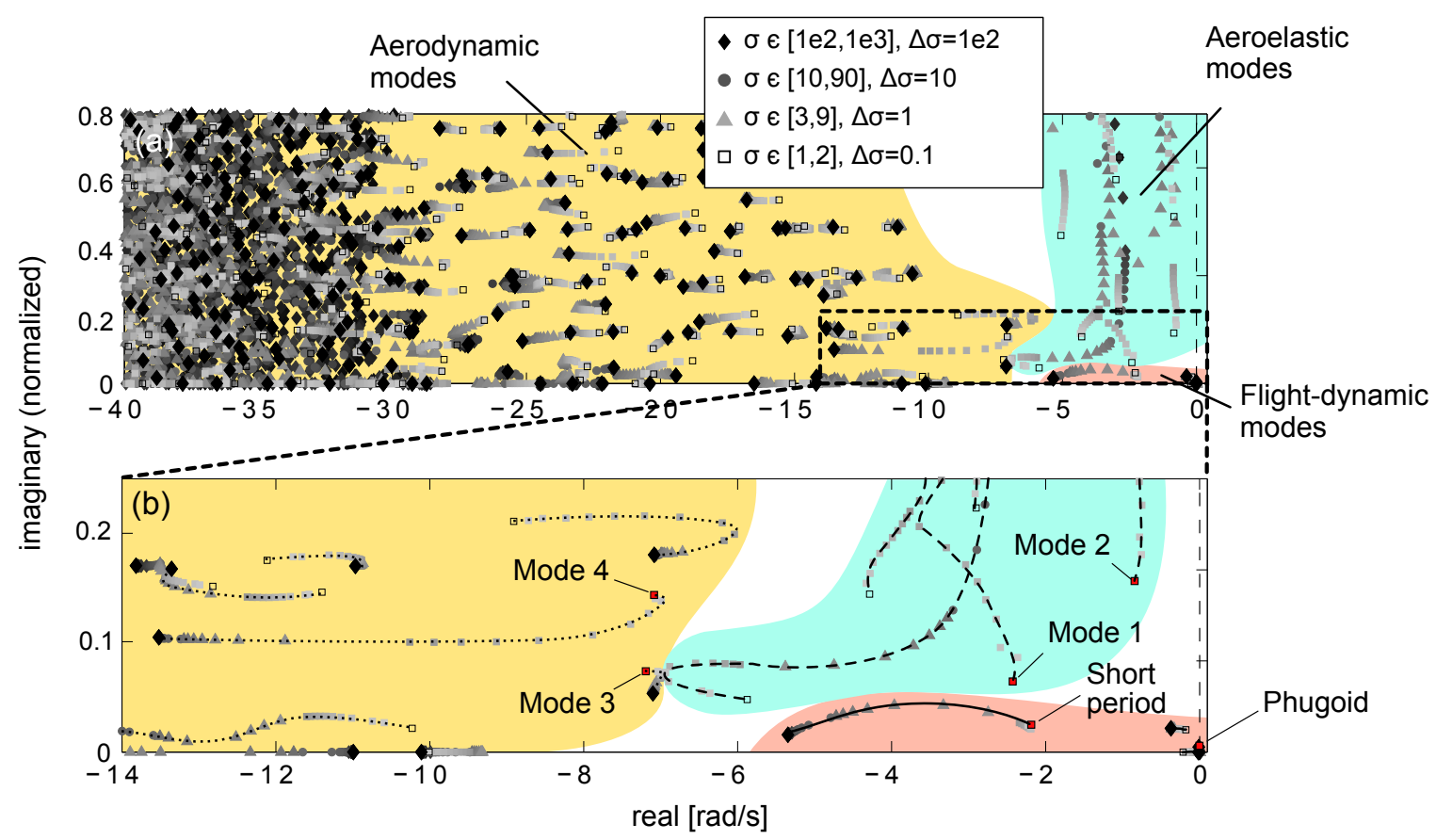

Figure 9: Root locus of the eigenvalue analysis with the stiffness of the main wing, $\sigma$, as parameter: flight dynamic, aeroelastic, and aerodynamic modes highlighted with solid, dashed, and dotted curves. Eigenvalues have been converted to continuous time and imaginary part normalized with $c /(2 \mathrm{~V})$.

The zoom in Figure 9(b) shows the frequency and damping overlap between the aerodynamic, aeroelastic and flight dynamic modes, where the variation with $\sigma$ has been traced with dotted, dashed and solid curves, respectively. We will examine six modes, which are highlighted in Figure 9(b) and correspond to the most flexible configuration with $\sigma=1$ ( $42 \%$ tip deflection). Only the aerodynamic component in the eigenvector will be investigated. The first two coupled modes have been designated by the names of their rigid-aircraft 
counterparts: phugoid and short period. Two aeroelastic modes have also been highlighted, which exhibit symmetric (Mode 1) and antisymmetric (Mode 2) bending/torsion couplings, and finally the symmetric (Mode 3) and antisymmetric (Mode 4) unsteady aerodynamic modes.

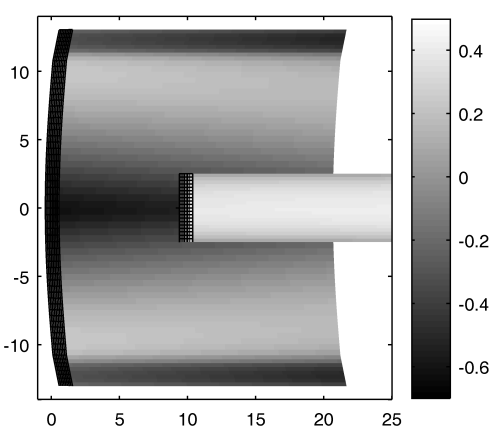

(a) Phugoid

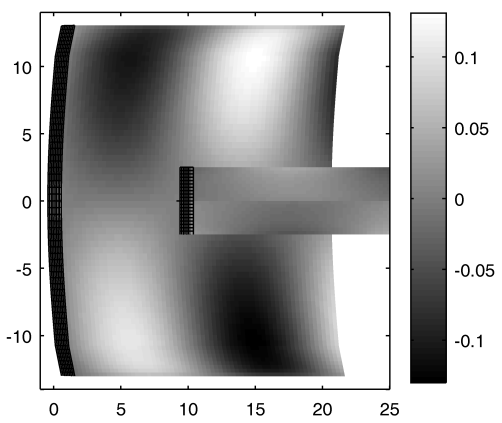

(d) Mode 2

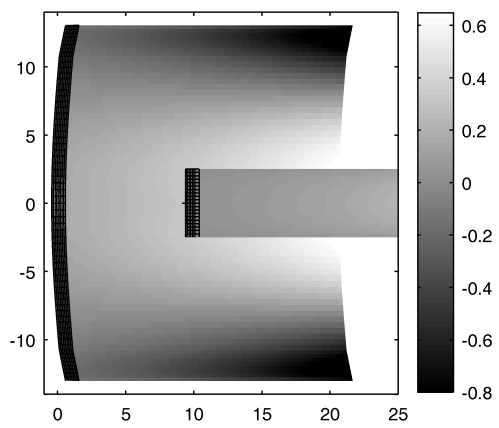

(b) Short period

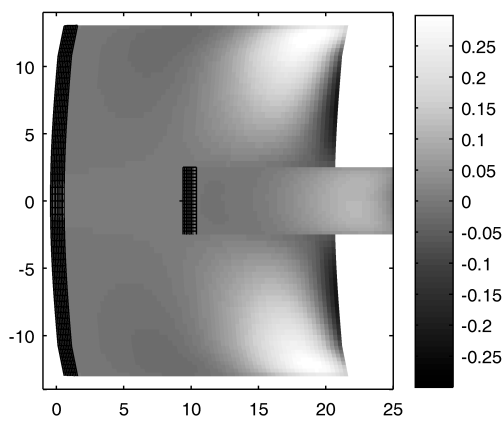

(e) Mode 3

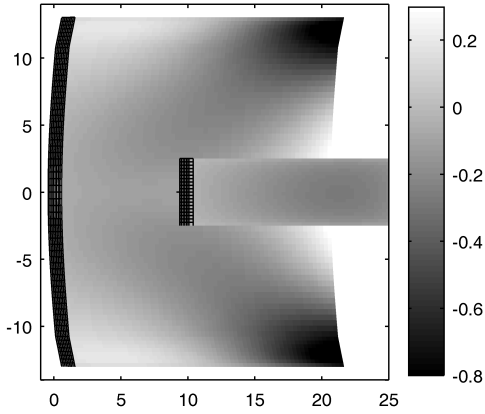

(c) Mode 1

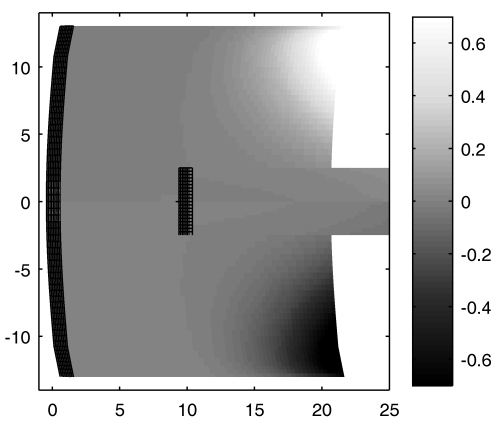

(f) Mode 4

Figure 10: Aerodynamic component of the mode shapes for a very flexible aircraft, $\sigma=1$, showing the circulation strengths of the vortex rings for the six modes highlighted in Figure 9(b).

Figure 10 shows the bound and wake circulation strengths, $\Gamma_{b}$ and $\Gamma_{w}$, of these mode shapes for the trimmed vehicle with $\sigma=1$. As one would expect, unsteady effects are negligible for the phugoid mode with no variation of circulation in the chordwise direction of the wake. With increasing frequency, however, quasi-steady models may fail to predict the flight-dynamic response accurately, as shown for the short-period mode in Figure 10(b). The higher-frequency aeroelastic modes (Modes 1 and 2) show distinct variations in the wake, which demonstrates the effect of elastic deformations on the unsteady aerodynamic loads. On the other hand, Modes 3 and 4 represent unsteady aerodynamic modes which couple with the structural and rigid-body dynamics of the aircraft and are excited in the similar frequency range as their aeroelastic and flight-dynamic counterparts (albeit highly damped).

A suitable reduced-order model should then preserve the relevant information of the unsteady flow to predict the actual dynamic response of the aircraft. This shows that, depending on the frequency content of the excitation, a large number of aerodynamic states in addition to the dominant flight-dynamic and aeroelastic modes may be required to capture the prevalent physics of the problem, which will investigated next on a full aircraft application.

\section{IV.B.2. Model Reduction for a Maneuvering HALE Aircraft}

In this section we will demonstrate the balanced truncation method, as outlined in Figure 2, to obtain the unsteady aerodynamic loads of the flexible HALE aircraft subject to commanded antisymmetric aileron inputs. The open-loop response of the maneuvering vehicle will be modeled using the coupled aeroelastic framework presented in Section II.C.2. Under the assumption of small displacements, the generalized unsteady aerodynamic loads are computed in time domain using the linearized UVLM. The inputs to the linear aerodynamic system are defined in Eq. (11) as the structural and rigid-body displacements in generalized coordinates, $u_{\Phi}$, and the aerodynamic inputs, $u_{A}$, to account for control surface deflections and atmospheric 
disturbances.

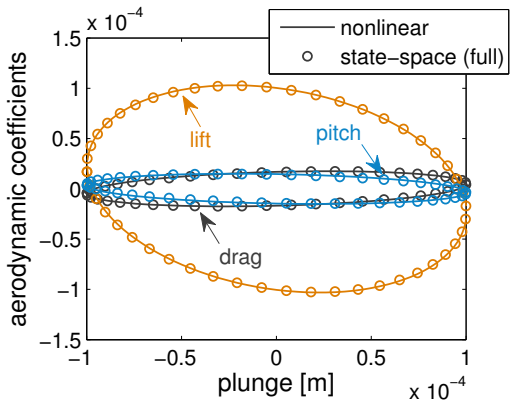

(a) Plunging motion

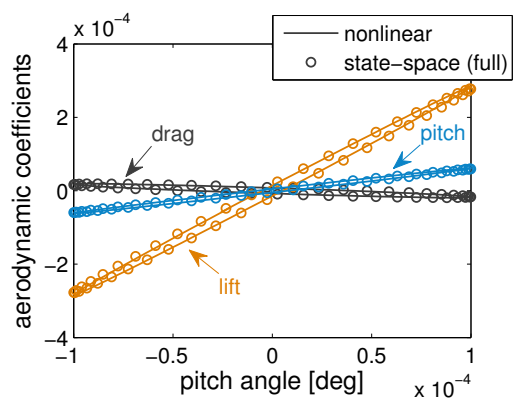

(b) Full-chord control surface deflection

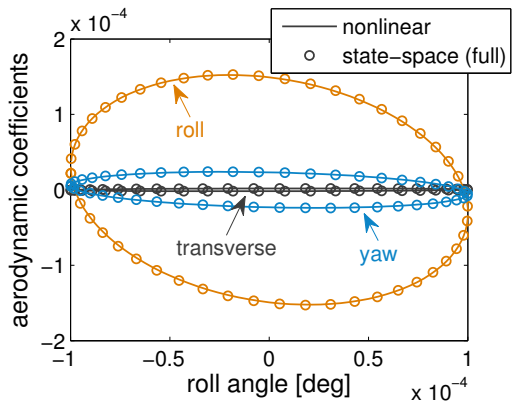

(c) Rolling motion

Figure 11: Aerodynamic coefficients for the flexible HALE wing $(\sigma=5$, no dihedral, and 5 deg AoA) subject to forced harmonic rigid-body motions with $\omega=2 \pi \mathrm{rad} / \mathrm{s}$ : comparison between the geometrically nonlinear time-marching and the linearized full-order state-space solutions.

First, the linearization of the UVLM is verified for a subproblem of the HALE configuration. The flexible main wing with $\sigma=5$ and zero-degree dihedral angle of the winglets is subjected to prescribed harmonic motions of the origin of the $A$ frame fixed to the wing root, as shown in Figure 8 . Sinusoidal plunge, pitch, and roll perturbations with an excitation frequency of $\omega=2 \pi \mathrm{rad} / \mathrm{s}$ (reduced frequency of $k \approx 0.1$ ) are prescribed with respect to the inertial frame of reference around a geometrically nonlinear equilibrium condition at $5 \mathrm{deg}$ angle of attack. This problem allows the verification of the aerodynamic load calculation of the aeroelastic framework, Eq. (11), where plunging and rolling motions of the flexible wing are prescribed maneuvers through the input $u_{\Phi}$ and the pitching motion is inputted through $u_{A}$ to verify a full-chord, fullspan control surface deflection. Comparison of the dominant aerodynamic coefficients with the nonlinear time-marching solution of the UVLM in Figure 11, demonstrates the validity of the linearized full-order state-space approach to predict the 3D unsteady aerodynamic loads. Note that, following the definition of the lift and pitching moment coefficients, the aerodynamic forces and moments have been normalized with the dynamic pressure, $\left(0.5 \rho V_{\infty}\right)$, and the total wing area of $32 \mathrm{~m}^{2}$. The resultant moments have also been normalized with the wing chord to obtain the aerodynamic moment coefficients.

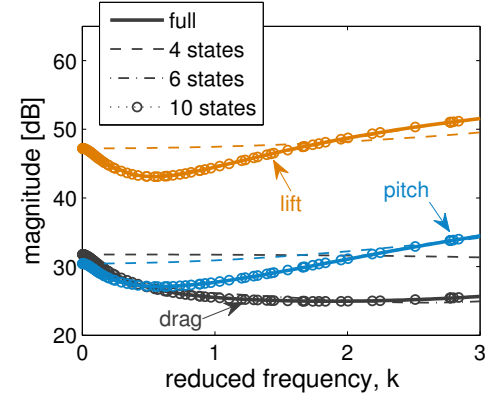

(a) Plunging motion

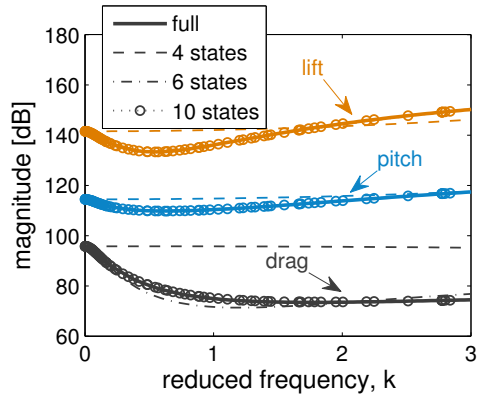

(b) Full-chord control surface deflection

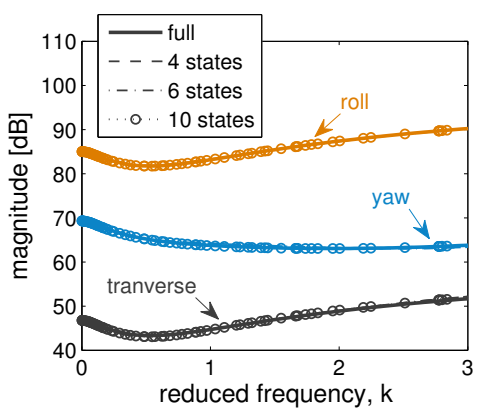

(c) Rolling motion

Figure 12: Comparison of reduced-order models in terms of frequency response of flexible HALE wing $(\sigma=5)$ with aerodynamic loads due to the harmonic plunging, pitching and rolling motions for different reduced frequencies, $k=\omega c /\left(2 V_{\infty}\right)$.

Following the reduced-order modeling approach introduced in Section III, we now focus on reducing the order of the linear aerodynamic system for the above HALE wing subject to prescribed harmonic motions. For the converged solution, the size of the full-order aerodynamic system reaches 4864 states due to the fine discretization of the wake for a time step of $0.02 \mathrm{~s}$ (and the same wing and wake discretization as in Section IV.B.1). However, wake panels that have converged far downstream may have only a small contribution on the unsteady aerodynamic loads which provides the motivation for model reduction. Figure 12 demonstrates the potential of balanced truncation on the large multi-input, multi-output aerodynamic system, which maps the 774 structural and rigid-body inputs to 390 aerodynamic load outputs. It shows the frequency 
response of different reduced-order aerodynamic systems for the above harmonic plunging, pitching and rolling motions and the corresponding dominant unsteady aerodynamic loads as outputs over a range of reduced frequencies, $k=\omega c /\left(2 V_{\infty}\right)$. The number of retained balanced states were again chosen according to the HSV of the balanced system (not shown here) and the results in Figure 12 suggest that as little as 6 aerodynamic states (from the original 4864 states) are sufficient to compute the unsteady loads of the flexible wing subject to rigid-body motions and control surface deflections even at high reduced frequencies. Even though we were able to reduce the size of the linear aerodynamic system dramatically, the flexiblebody dynamics DoF still dictate the large number of inputs (and outputs) of the reduced-order aerodynamic system.

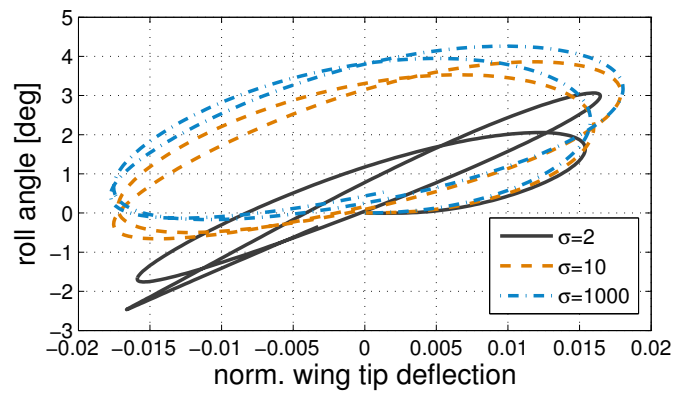

Figure 13: Open-loop response of HALE aircraft subject to antisymmetric aileron inputs for the full-order state-space system for varying stiffness, $\sigma$. Tip deflections are normalized with $B \cdot \sigma^{-1}$.

At last, we will also demonstrate modal reduction of the nonlinear flexible-body dynamics EoM, as outlined in Section II.B.1, on the open-loop response of the full HALE configuration subject to antisymmetric, sinusoidal aileron inputs with an excitation frequency of $1.29 \mathrm{rad} / \mathrm{s}$ and amplitude of $5 \mathrm{deg}$ (comparable to Dutch roll in Table 4). The resulting full-order open-loop response is presented in Figure 13 in terms of the correlation between the rigid-body response (roll angle of the aircraft) and the structural dynamics behavior (deflection of the right wing tip normalized with the semi-span $B$ and the stiffness parameter, $\sigma$ ). The transient response is shown for three cases of $\sigma$, where $\sigma=1000$ corresponds to the near-rigid configuration. Even though the aileron excitation results in small structural deformations below 1\%, in addition to the trim deformations, the effect on the rigid-body response is significant. While the near-rigid configuration always returns to its initial roll attitude, the flexible aircraft configurations overshoot instead and thus amplify the gyroscopic coupling between the (stable) structural and flight dynamics.

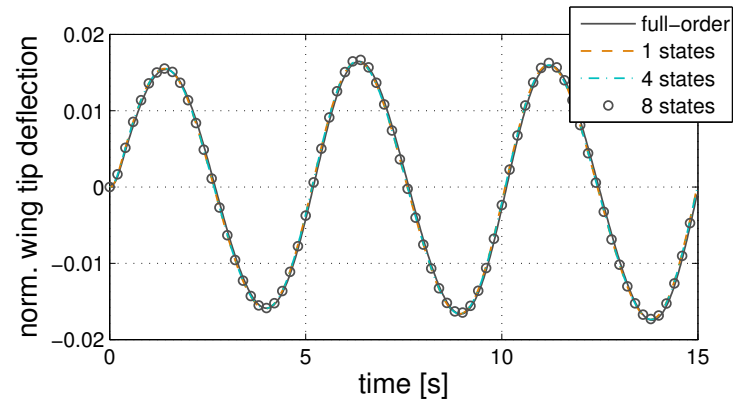

(a) Vertical tip deflection normalized with $B \cdot \sigma^{-1}$

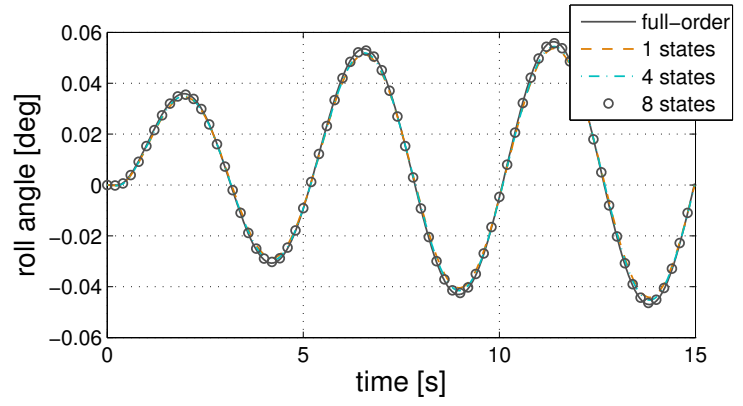

(b) Aircraft roll response

Figure 14: Effect of model order of aerodynamic system on open-loop response of HALE for $\sigma=2$ and all flexible-body dynamics modes.

The aerodynamic subsystem and the modal form of the flexible-body dynamics module of the nonlinear coupled aeroelastic model, as outlined in Figure 1, will now be reduced separately through balanced and modal truncation, respectively. Model reduction of the large aerodynamic system, shown in Figure 14 for the roll response of the aircraft and the resulting elastic deformations, illustrates that only one (balanced) aerodynamic state is sufficient to represent the complex flow features of the full-order aerodynamic model with over 3000 states. More states are required to resolve the unsteady effects of the control surface deployment. This is also reflected in Figure 15, which shows the time history of the wake of the maneuvering aircraft for 


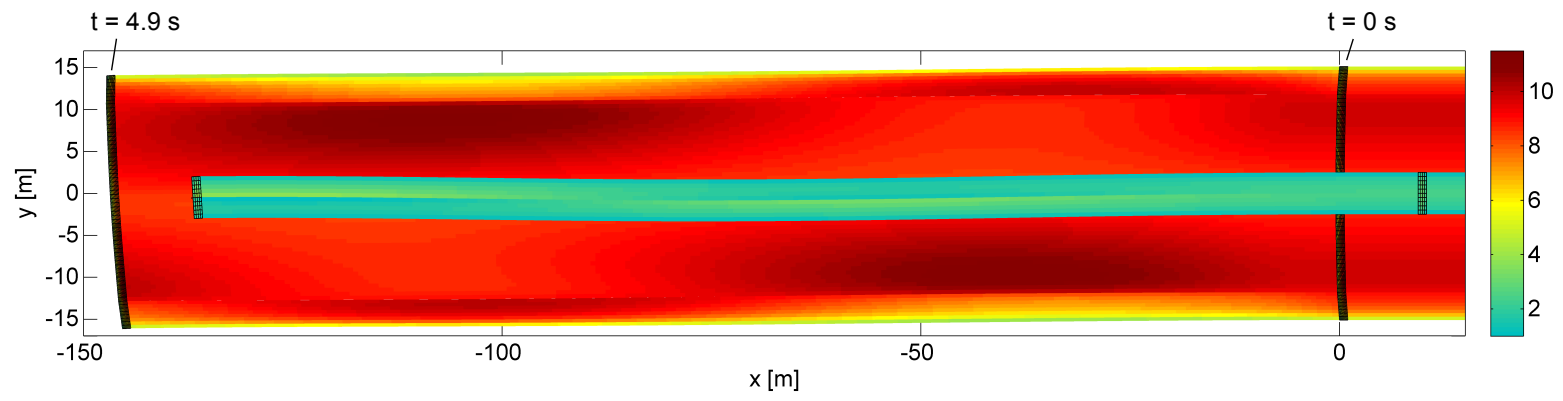

(a) Full-order aerodynamic model with 3120 states

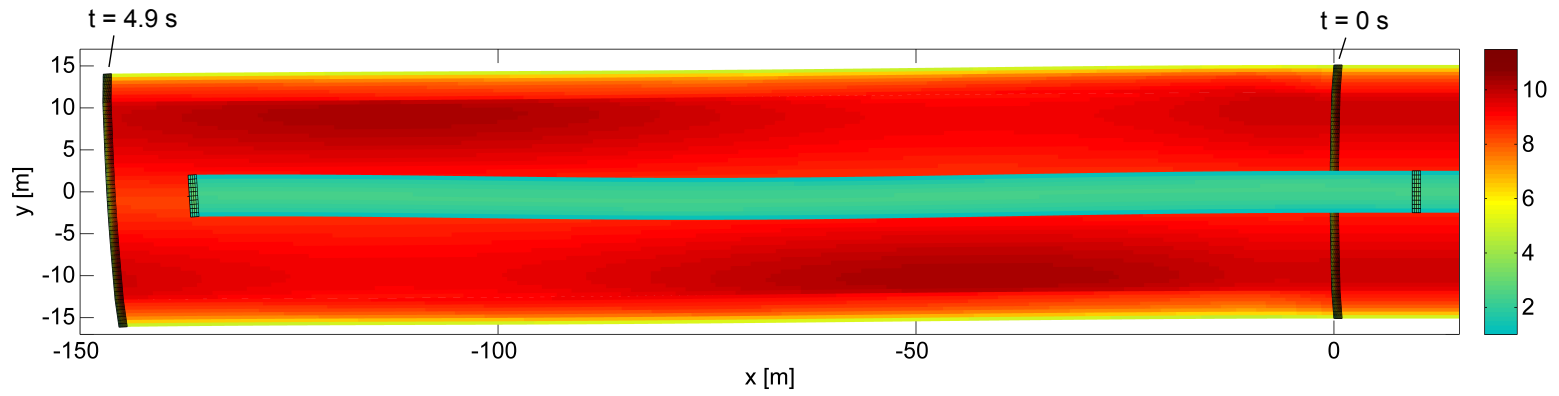

(b) Reduced-order balanced system with four aerodynamic states

Figure 15: History of the wake for the maneuvering flexible aircraft with $\sigma=2$ and subject to aileron inputs, showing the circulation strengths of the wake vortex rings, $\Gamma_{w}$.

the first period of the antisymmetric aileron input, $0 \leq t \leq 4.9 \mathrm{~s}$. Note that in the actual simulation the wake is truncated at 15 chord-lengths and Figure 15 instead presents the evolution of circulation strength of the trailing edge vortex panels as they are shed into the wake at each time step. The results of the reduced-order system have been retrieved through reverse transformation of the truncated balanced state vector. Comparison with the full-order model shows that variations in the flow due to the aircraft motion are captured accurately despite the tremendous reduction of the aerodynamic model, but the reduced-order model fails to capture the aerodynamics around the deployed control surfaces in the winglets of the main wing.

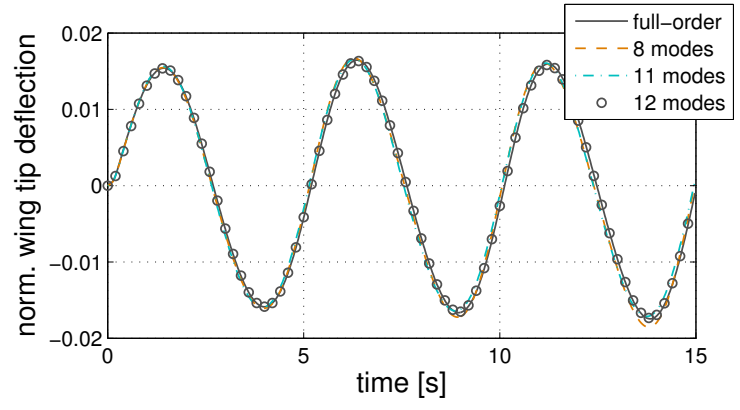

(a) Vertical tip deflection normalized with $B \cdot \sigma^{-1}$

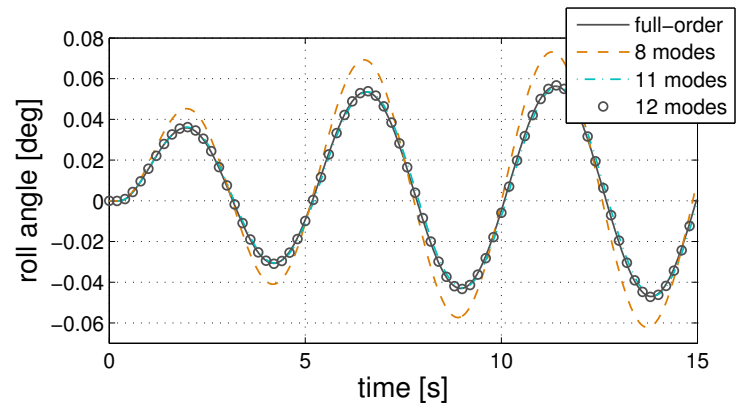

(b) Aircraft roll response

Figure 16: Effect of number of flexible-body dynamics modes (ascending order of frequency) on open-loop response of HALE for $\sigma=2$ and full-order aerodynamic system.

Next, we reduce the number of inputs and outputs of the aerodynamic system through modal truncation of the flexible-body dynamics model, shown in 16 for the full-order aerodynamic system. Note that the truncation includes all modes in ascending order of the vibration frequency, even though it is clear from the antisymmetric nature of the excitation that antisymmetric bending/torsion modes predominantly contribute to the elastic response of the aircraft. The first antisymmetric bending mode (mode 8), in addition to the six rigid-body modes, is sufficient to capture the bending response of the aircraft, as shown in Figure 16(a), 
but the vehicle roll response in Figure 16(b) is strongly affected by the twisting of the main wing due to the aileron excitation. Hence, the first antisymmetric torsion mode (mode 11) is also required to fully capture the dominant dynamics of the HALE aircraft. Both structural modes of the unconstrained aircraft have been depicted in Figure 17 for illustration.

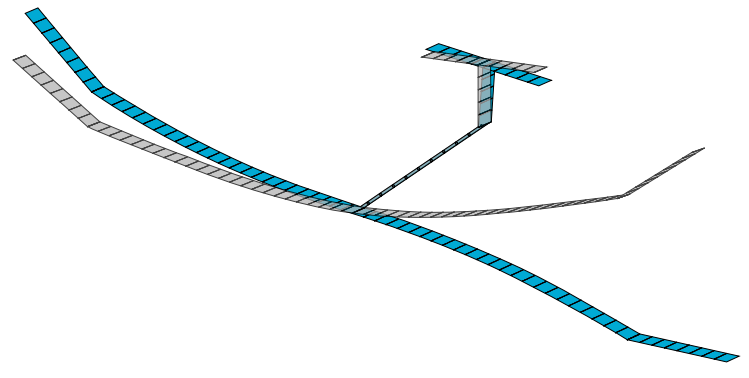

(a) $12.97 \mathrm{rad} / \mathrm{s}$

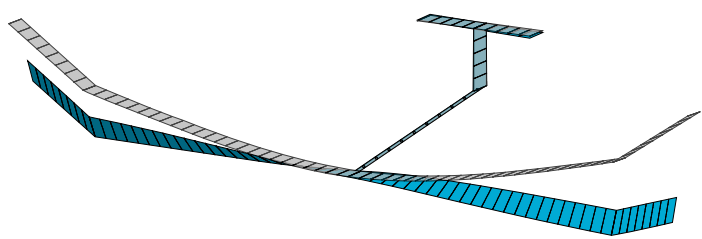

(b) $24.78 \mathrm{rad} / \mathrm{s}$

Figure 17: First antisymmetric (a) bending and (b) torsion structural modes of the flexible HALE configuration $(\sigma=2)$ around trimmed flight condition (trim geometry superimposed in gray).

Hence, only eight flexible-body modes (two structural and six rigid-body modes) and a single aerodynamic state are required to adequately model the lateral dynamics of the maneuvering aircraft but, naturally, the elastic response is captured more accurately if more higher-frequency modes are included in the projection of the flexible-body dynamics EoM. This can be seen from Figure 18, which shows the $L_{\infty}$ error of the elastic (tip deflection) and rigid-body (roll angle) response for the three HALE configurations as presented in Figure 13, where the maximum of both error norms is shown. The relative error, which is normalized with the maximum wing deflection and roll angle for each $\sigma$, respectively, shows the contribution of the elastic modes and balanced aerodynamic states on the accuracy of the reduced-order system response. For the most flexible configuration in Figure 18(a), the antisymmetric bending and torsion modes $(8,11,12,15,18)$ clearly dominate the response but, as expected, the number of required elastic modes reduces significantly for the stiffer aircraft in Figures 18(b)-(c).

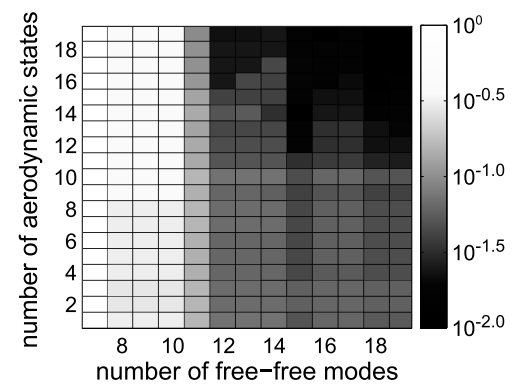

(a) $\sigma=2$

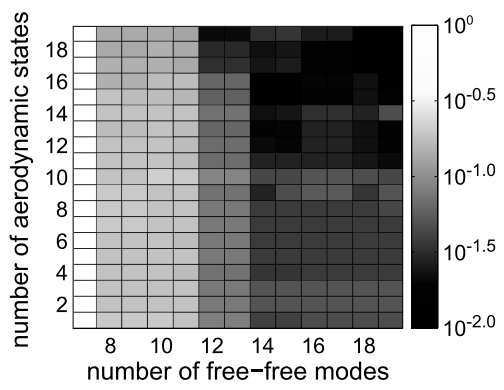

(b) $\sigma=10$

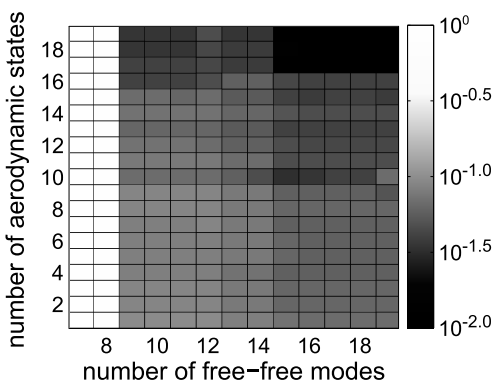

(c) $\sigma=1000$

Figure 18: Relative $L_{\infty}$ error of the HALE aircraft reduced-order open-loop response for varying number of aerodynamic states and free-free modes (including the six rigid-body modes) and three values of the stiffness parameter, $\sigma$.

Increasing the number of free-free modes in the projection of the flexible-body dynamics EoM may increase accuracy of the higher frequency response, but it can also deteriorate the effectiveness of balanced truncation of the aerodynamic system. This is evident in Figure 18(a), where the inclusion of unnecessary symmetric elastic modes, e.g. modes 13-14 and 16-17, harms the accuracy of the reduced-order models. The symmetric modes contribute little to the system response, but increase the number of inputs and outputs of the aerodynamic system and more aerodynamic states are required to restore accuracy. This is an important aspect, if the proposed aeroelastic reduced-order modeling approach were to be used for control synthesis and/or optimization.

In summary, the model reduction approach produces very low-order aeroelastic models which can still accurately predict the dynamic response of flexible maneuvering aircraft. This is done for very flexible 
configurations with geometrically nonlinear wing deformations at trimmed flight. The results in Figure 18 show that the full-order aerodynamic system can be reduced dramatically (by three orders of magnitude) and still captures the inherent 3D features of the unsteady flow which are tightly coupled with the flexible-body dynamic response of the aircraft.

\section{Conclusion}

This paper has presented a model reduction approach, using the balanced truncation method, on the coupled flight dynamics and aeroelastic response of maneuvering flexible aircraft with large (geometricallynonlinear) static trim deformations. Under the assumption of small wing deformations in the subsequent system dynamics, the unsteady aerodynamic loads were obtained in time domain using the three-dimensional unsteady vortex lattice method, which was linearized around the trimmed flight condition with a deformed aerodynamic lattice and force-free wakes in the steady state. Consistent linearization of the structural degrees of freedom allowed the nonlinear flexible-body dynamics to be projected onto a small number of dominant vibration modes of the unconstrained structure. While this modal approach accounts for the nonlinearity in the rigid-body dynamics equations due to large vehicle maneuvers and preserves all gyroscopic couplings between rigid-body and structural dynamics, it reduces the number of generalized structural coordinates and aerodynamic loads - the inputs and outputs of the aerodynamic system - which has been shown to improve the effectiveness of balanced model reduction of the linear aerodynamic subsystem.

The proposed model reduction method has been exercised on a representative HALE configuration subject to commanded antisymmetric control surface inputs. A linear stability analysis of the flexible aircraft demonstrated the effect of flexibility on the vehicle flight-dynamic, aeroelastic, and aerodynamic modes which provided an insight into the effect of flow unsteadiness on the aircraft response and the validity of quasisteady models to capture the aerodynamics of maneuvering aircraft. Subsequent balanced truncation of the linear aerodynamic system was demonstrated on the open-loop response of the vehicle, which highlighted the potential of balancing methods with dramatic model reductions by three orders of magnitude while the inherent three-dimensional features of the unsteady flow are preserved. Projection of the flexible-body degrees of freedom onto a few vibration modes not only provided further model reduction but was also shown to improve effectiveness of the balanced truncation of the aerodynamic system for a very flexible aircraft configuration. Model reduction of an unstable aeroelastic system was further exercised for the synthesis of a robust flutter suppression system using $\mathcal{H}_{\infty}$ control, which was demonstrated for the Goland wing problem at $7 \%$ above the flutter velocity. A controller based on only two balanced states performed best to stabilize the aeroelastic response.

In summary, the proposed balanced model reduction approach of the aeroelastic framework provides a powerful tool to simulate the dynamics of highly-efficient very flexible aircraft with a similar computational cost of linear frequency approaches based on the doublet lattice method. The proposed method however removes kinematic restrictions and obtains the aerodynamic loads directly in time domain, which makes it also ideal for control synthesis and optimization. Moreover, even though the aeroelastic framework is projected onto a few vibration modes of the unconstrained structure, it accounts for the inertial coupling between the vehicle structural response and the nonlinear rigid-body dynamics.

\section{Acknowledgments}

The work of Henrik Hesse is sponsored by the UK Engineering and Physical Sciences Research Council (EPSRC). This support is gratefully acknowledged.

\section{References}

\footnotetext{
${ }^{1}$ Albano, E. and Rodden, W. P., "A Doublet-Lattice Method for Calculating Lift Distributions on Oscillating Surfaces in Subsonic Flow," AIAA Journal, Vol. 7, No. 2, 1969, pp. 279-285.

${ }^{2}$ Karpel, M., "Procedures and Models for Aeroservoelastic Analysis and Design," ZAMM - Journal of Applied Mathematics and Mechanics / Zeitschrift fr Angewandte Mathematik und Mechanik, Vol. 81, No. 9, 2001, pp. 579592.

${ }^{3}$ Wright, J. R. and Cooper, J. E., Introduction to Aircraft Aeroelasticity and Loads, John Wiley \& Sons Ltd, Chichester, UK, 2008.

${ }^{4}$ Murua, J., Palacios, R., and Graham, J., "Applications of the unsteady vortex-lattice method in aircraft aeroelasticity and flight dynamics," Progress in Aerospace Sciences, Vol. 55, Nov. 2012, pp. 46-72.
} 
${ }^{5}$ Schmidt, D. K., Modern Flight Dynamics, McGraw-Hill, 2011.

${ }^{6}$ Hesse, H. and Palacios, R., "Consistent structural linearisation in flexible-body dynamics with large rigid-body motion," Computers \& Structures, Vol. 110-111, 2012, pp. 1-14.

${ }^{7}$ Hesse, H., Palacios, R., and Murua, J., "Consistent Structural Linearization in Flexible Aircraft Dynamics with Large Rigid-Body Motion," AIAA Journal, [Journal version of AIAA 2012-1402, under review].

${ }^{8}$ Hall, K., "Eigenanalysis of Unsteady Flows about Airfoils, Cascades and Wings," AIAA Journal, Vol. 32, No. 12, 1994, pp. $2426-2432$.

${ }^{9}$ Loeve, M., Probability theory, Van Nostrand, New York, USA, 1955.

${ }^{10}$ Berkooz, G., Holmes, P., and Lumley, J. L., "The Proper Orthogonal Decomposition in the Analysis of Turbulent Flows," Annual Review of Fluid Mechanics, Vol. 25, No. 1, 1993, pp. 539-575.

${ }^{11}$ Romanowski, M. C., "Reduced order unsteady aerodynamic and aeroelastic models using Karhunen-Loeve eigenmodes," Proceedings of the Sixth AIAA Symposium on Multidisciplinary Analysis and Optimization, Bellevue, WA, USA, 1996.

${ }^{12}$ Dowell, E. H., Hall, K. C., and Romanowski, M. C., "Eigenmode analysis in unsteady aerodynamics: reduced order models," Applied Mechanics Reviews, Vol. 50, 1997, pp. 371.

${ }^{13}$ Lucia, D. J., Beran, P. S., and Silva, W. A., "Reduced-order modeling: new approaches for computational physics," Progress in Aerospace Sciences, Vol. 40, No. 12, 2004, pp. 51-117.

${ }^{14}$ Rule, J., Clark, R., and Cox, D., "Aerodynamic Model Reduction Through Balanced Realization," AIAA Journal, Vol. 42, No. 5, 2004, pp. 1045-1048.

${ }^{15}$ Moore, B., "Principal component analysis in linear systems: Controllability, observability, and model reduction," IEEE Transactions on Automatic Control, Vol. 26, No. 1, Feb. 1981, pp. 17-32.

${ }^{16}$ Glover, K., "All optimal Hankel-norm approximations of linear multivariable systems and their $L^{\infty}$-error bounds," International Journal of Control, Vol. 39, No. 6, 1984, pp. 1115-1193.

${ }^{17}$ Mauermann, T., Flexible Aircraft Modelling for Flight Loads Analysis of Wake Vortex Encounters, Ph.D. thesis, Technische Universität Carolo-Wilhelmina zu Braunschweig, Braunschweig, Germany, 2011.

${ }^{18}$ Willcox, K. and Peraire, J., "Balanced Model Reduction via the Proper Orthogonal Decomposition," AIAA Journal, Vol. 40, No. 11, 2002, pp. 2323-2330.

${ }^{19}$ Haghighat, S., Martins, J. R. R. A., and Liu, H. H. T., "Model-Predictive Gust Load Alleviation Controller for a Highly Flexible Aircraft," Journal of Guidance, Control, and Dynamics, Vol. 35, No. 6, 2012, pp. 1751-1766.

${ }^{20}$ Murua, J., Palacios, R., and Graham, J. M. R., "Open-Loop Stability and Closed-Loop Gust Alleviation on Flexible Aircraft Including Wake Modeling," $53^{\text {rd }}$ AIAA/ASME/ASCE/AHS/ASC Structures, Structural Dynamics, and Materials Conference, AIAA 2012-1484, Honolulu, HI, USA, 2012.

${ }^{21}$ Murua, J., Palacios, R., and Graham, J. M. R., "Assessment of Wake-Tail Interference Effects on the Dynamics of Flexible Aircraft," AIAA Journal, Vol. 50, No. 7, July 2012, pp. 1575-1585.

${ }^{22}$ Simo, J. C. and Vu-Quoc, L., "On the dynamics in space of rods undergoing large motions - A geometrically exact approach," Computer Methods in Applied Mechanics and Engineering, Vol. 66, No. 2, 1988, pp. 125-161.

${ }^{23}$ Géradin, M. and Cardona, A., Flexible multibody dynamics: a finite element approach, John Wiley \& Sons Ltd, Chichester, UK, 2001.

${ }^{24}$ Stevens, B. L. and Lewis, F. L., Aircraft Control and Simulation, John Wiley \& Sons, Inc., New York, NY, USA, 1992.

${ }^{25}$ Géradin, M. and Rixen, D., Mechanical Vibrations: Theory and Application to Structural Dynamics, John Wiley \& Sons Ltd, Chichester, UK, 2nd ed., 1997.

${ }^{26}$ Palacios, R., Murua, J., and Cook, R., "Structural and Aerodynamic Models in the Nonlinear Flight Dynamics of Very Flexible Aircraft," AIAA Journal, Vol. 48, No. 11, 2010, pp. 2648-2559.

${ }^{27}$ Amsallem, D. and Farhat, C., "An Online Method for Interpolating Linear Parametric Reduced-Order Models," SIAM Journal on Scientific Computing, Vol. 33, No. 5, Jan. 2011, pp. 2169-2198.

${ }^{28}$ Craig, R. R., Structural dynamics: An Introduction to Computer Methods, John Wiley \& Sons, Inc., New York, USA, 1981.

${ }^{29}$ Safonov, M. and Chiang, R., "A Schur method for balanced-truncation model reduction," IEEE Transactions on Automatic Control, Vol. 34, No. 7, July 1989, pp. $729-733$.

${ }^{30}$ Meyer, D. G., "A Fractional Approach to Model Reduction," American Control Conference, 1988, June 1988, pp. 1041 -1047 .

${ }^{31}$ Balas, G., Chiang, R., Packard, A., and Safonov, M., Robust Control Toolbox Users Guide, The MathWorks, Inc., Natick, MA, USA, 2006.

${ }^{32}$ Benner, P. and Quintana-Ortí, E. S., "Model Reduction Based on Spectral Projection Methods," Dimension Reduction of Large-Scale Systems, edited by P. Benner, D. C. Sorensen, V. Mehrmann, T. J. Barth, M. Griebel, D. E. Keyes, R. M. Nieminen, D. Roose, and T. Schlick, Vol. 45 of Lecture Notes in Computational Science and Engineering, Springer Berlin Heidelberg, 2005, pp. 5-48.

${ }^{33}$ Skogestad, S. and Postlethwaite, I., Multivariable feedback control: analysis and design, John Wiley \& Sons Ltd, Chichester, UK, 2005.

${ }^{34}$ Laub, A., Heath, M., Paige, C., and Ward, R., "Computation of system balancing transformations and other applications of simultaneous diagonalization algorithms," IEEE Transactions on Automatic Control, Vol. 32, No. 2, Feb. 1987, pp. 115-122.

${ }^{35}$ Hammarling, S. J., "Numerical Solution of the Stable, Non-negative Definite Lyapunov Equation Lyapunov Equation," IMA Journal of Numerical Analysis, Vol. 2, No. 3, Jan. 1982, pp. 303-323.

${ }^{36}$ Enns, D., Model reduction for control system design, Ph.D. thesis, Stanford University, Department of Aeronautics and Aerospace, 1984.

${ }^{37}$ Vidyasagar, M., Control System Synthesis: A Factorization Approach, MIT Press, 1987. 
${ }^{38}$ Meyer, D. and Franklin, G., "A connection between normalized coprime factorizations and linear quadratic regulator theory," IEEE Transactions on Automatic Control, Vol. 32, No. 3, March 1987, pp. 227-228.

${ }^{39}$ Meyer, D., "The construction of special coprime factorizations in discrete time," IEEE Transactions on Automatic Control, Vol. 35, No. 5, May 1990, pp. $588-590$.

${ }^{40}$ Vidyasagar, M., "Normalised coprime factorizations for nonstrictly proper systems," IEEE Transactions on Automatic Control, Vol. 33, No. 3, March 1988, pp. $300-301$.

${ }^{41}$ Goland, M., "The flutter of a uniform cantilevered wing," Journal of Applied Mechanics, Vol. 12, No. 4, 1945, pp. A197A198.

${ }^{42}$ Patil, M. J., Hodges, D., and Cesnik, C., "Nonlinear Aeroelastic Analysis of Complete Aircraft in Subsonic Flow," Journal of Aircraft, Vol. 37, No. 5, 2000, pp. 753-760.

${ }^{43}$ Wang, Z., Chen, P. C., Liu, D. D., and Mook, D. T., "Nonlinear-Aerodynamics/Nonlinear-Structure Interaction Methodology for a High-Altitude Long-Endurance Wing," Journal of Aircraft, Vol. 47, No. 2, 2010, pp. 556-566.

${ }^{44}$ Katz, J. and Plotkin, A., Low-Speed Aerodynamics, Cambridge Aerospace Series, Cambridge University Press, New York, NY, USA, 2nd ed., 2001.

${ }^{45}$ Zhou, K., Doyle, J. C., and Glover, K., Robust and Optimal Control, Prentice-Hall, Upper Saddle River, 1 st ed., 1996.

${ }^{46}$ Vinnicombe, G., "The robustness of feedback systems with bounded complexity controllers," Automatic Control, IEEE Transactions on, Vol. 41, No. 6, 1996, pp. $795-803$.

${ }^{47}$ Cook, R. G., Palacios, R., and Goulart, P., "Robust Gust Alleviation and Stabilization of Very Flexible Aircraft," AIAA Journal, Vol. 51, No. 2, February 2013, pp. 330-340.

${ }^{48} \mathrm{Ng}$, B. F., Palacios, R., Graham, J., and Kerrigan, E. C., "Robust control synthesis for gust load alleviation from large aeroelastic models with relaxation of spatial discretisation," Proceedings of the EWEA 2012, Copenhagen, Denmark, 2012.

${ }^{49}$ Patil, M. J., Hodges, D. H., and Cesnik, C. E., "Nonlinear aeroelasticity and flight dynamics of high-altitude longendurance aircraft," Journal of Aircraft, Vol. 38, No. 1, 2001, pp. 88-94. 